\title{
The Need to Pair Molecular Monitoring Devices with Molecular Imaging to Personalize Health
}

\author{
Zachary J. Comeau ${ }^{1,2}$, Benoît H. Lessard ${ }^{1,3}$, and Adam J. Shuhendler ${ }^{2,4,5}$ \\ ${ }^{1}$ Department of Chemical and Biological Engineering, University of Ottawa, 161 Louis Pasteur, Ottawa, ON K1N 6N5, Canada \\ ${ }^{2}$ Department of Chemistry and Biomolecular Sciences, University of Ottawa, 150 Louis Pasteur, Ottawa, ON K1N 6N5, Canada \\ ${ }^{3}$ School of Electrical Engineering and Computer Science, University of Ottawa, 800 King Edward Ave., Ottawa, ON K1N 6N5, Canada \\ ${ }^{4}$ Department of Biology, University of Ottawa, 30 Marie Curie, Ottawa, ON K1N 6N5, Canada \\ ${ }^{5}$ University of Ottawa Heart Institute, 40 Ruskin St, Ottawa, ON K1Y 4W7, Canada 2022
}

\begin{abstract}
By enabling the non-invasive monitoring and quantification of biomolecular processes, molecular imaging has dramatically improved our understanding of disease. In recent years, non-invasive access to the molecular drivers of health versus disease has emboldened the goal of precision health, which draws on concepts borrowed from process monitoring in engineering, wherein hundreds of sensors can be employed to develop a model which can be used to preventatively detect and diagnose problems. In translating this monitoring regime from inanimate machines to human beings, precision health posits that continual and on-the-spot monitoring are the next frontiers in molecular medicine. Early biomarker detection and clinical intervention improves individual outcomes and reduces the societal cost of treating chronic and late-stage diseases. However, in current clinical settings, methods of disease diagnoses and monitoring are typically intermittent, based on imprecise risk factors, or self-administered, making optimization of individual patient outcomes an ongoing challenge. Low-cost molecular monitoring devices capable of on-the-spot biomarker analysis at high frequencies, and even continuously, could alter this paradigm of therapy and disease prevention. When these devices are coupled with molecular imaging, they could work together to enable a complete picture of pathogenesis. To meet this need, an active area of research is the development of sensors capable of point-of-care diagnostic monitoring with an emphasis on clinical utility. However, a myriad of challenges must be met, foremost, an integration of the highly specialized molecular tools developed to understand and monitor the molecular causes of disease with clinically accessible techniques. Functioning on the principle of probe-analyte interactions yielding a transducible signal, probes enabling sensing and imaging significantly overlap in design considerations and targeting moieties, however differing in signal interpretation and readout. Integrating molecular sensors with molecular imaging can provide improved data on the personal biomarkers governing disease progression, furthering our understanding of pathogenesis, and providing a positive feedback loop toward identifying additional biomarkers and therapeutics. Coupling molecular imaging with molecular monitoring devices into the clinical paradigm is a key step toward achieving precision health.
\end{abstract}

Keywords Molecular monitoring $\cdot$ Molecular imaging $\cdot$ Molecular sensing $\cdot$ Precision health $\cdot$ Personalized medicine $\cdot$ Sensor design

Correspondence to: Adam J. Shuhendler; e-mail:

Adam.Shuhendler@uottawa.ca 


\section{Introduction}

The traditional approach to disease management centers around treating symptoms as they present, often as a onesize-fits-all approach for drug therapies and diagnostics, limiting preemptive interventions and clinical flexibility [1, 2]. Though modern disease diagnostics as a facet of precision medicine now better incorporate individual variability through the use of advanced molecular tools, disease screening and chronic disease management often involves rudimentary, sometimes self-administered, screenings based on heuristic risk factors [3]. However, diseases often present molecular biomarkers well before they present outward signs and symptoms, providing an opportunity for early disease diagnosis. Powerful methods, such as metabolomics, gene sequencing, and molecular imaging aided by modern computational tools have enabled us to begin to understand some of the biomarkers that signal disease onset, but practicable implementations have been an ongoing challenge [4, 5]. Nonetheless, an improved understanding of the human model, in addition to the societal and economic value of preventative medicine, has prompted a push toward achieving precision health. Described by Gambhir et al. (2018), the notion of precision health draws on the concepts of engine health monitoring, where a simulated digital twin, constructed with a huge volume of real-world sensor data, forecasts problems to enable preventative repairs. While modern imaging techniques are exponentially increasing the data available, pairing these techniques with continuous sensor data is required to complete the human model. Within clinical settings, molecular imaging, as a part of precision medicine, is being increasingly used to directly assay an individual's disease state. However, there are still large gaps of time where disease progression or treatment efficacy is largely unknown. Blood analysis or point-of-care tests can reduce the length of these gaps, providing additional data points to draw from for clinical assessments, but the fundamental paradigm remains unchanged. Furthermore, while physiological conditions, such as heart rate, blood $\mathrm{O}_{2}$, and blood pressure are monitored continuously, with parameters outside accepted limits triggering immediate intervention, this is still often a reactionary intervention within the clinical setting. Molecular monitoring devices able to incorporate additional metrics into this continuous monitoring umbrella could alter the clinical paradigm and, when paired with molecular imaging, could improve clinical assessments by providing a more complete picture of disease pathogenesis. Finally, whereas a jet engine is constantly surveilled in real-time by hundreds of sensors to prevent failure, individuals are encouraged to self-monitor, reporting symptoms as they present, both preventatively and as part of disease management. Accessible molecular sensors are necessary to enable earlier, more objective, and more precise monitoring outside of the clinical setting both pre- and post-diagnosis. By increasingly pairing molecular imaging techniques with molecular monitoring devices, the clinical paradigm can be reimagined as we complete the human model, shifting from reactive to preventative medicine and enabling precision health.

Clinical imaging, including computed tomography (CT), ultrasound (US), magnetic resonance imaging (MRI), and positron emission tomography (PET), among others, are well-established techniques that create a visual representation of the interior of the body. Each technique requires the input of energy into the body, where the energy is differentially modulated by specific body tissue types, and the resulting modulated output energy is captured using specialized detectors or cameras. As a specialized implementation, molecular imaging has been broadly defined as a technique to monitor molecular processes directly or indirectly through the visualization or characterization of biological processes at the molecular and cellular levels. While clinical imaging, including optical techniques (e.g., optical coherence tomography), can provide high-resolution anatomical information, accessing physiological changes at the molecular level has been shown to provide much earlier opportunities for disease diagnosis, monitoring disease progression, or tracking therapeutic outcomes. Such a level of interrogation is achieved through molecular imaging, which differs from traditional anatomical imaging often through the use of injectable imaging agents that bind to or interact with biomolecules of interest, enabling their noninvasive visualization to provide a more complete diagnostic picture for clinicians.

In our view, a molecular monitoring device is broadly defined by any molecular structure used for sensing an analyte where the interaction of device and analyte produces some detectable change. Changes can be transduced, and thus measured optically, by mass, electromagnetically, or electronically. Electronic biosensors are then any molecular sensor engineered to detect a biomolecular analyte and operates by converting the interaction of analyte with the sensor into an electronic signal. Thus, electronic biosensors at their most basic level operate in the same way as molecular imaging techniques: a probe-analyte interaction is transduced into measurable signal and as a result, both molecular imaging and electrical biosensors share many of the same probe design considerations. However, whereas molecular imaging probes are designed to target specific biological systems, biomarker availability guides biosensor design.

Biosensors can be broadly subdivided into two of four categories: single use versus continual; and invasive versus non-invasive. Single use sensors often involve a destructive or covalent binding process, wherein a sample is collected, often invasively, from the patient and introduced to the sensor. Traditional biochemical techniques would fit this definition, however, as they require specific instruments and professional operators their point-of-care applications are limited. Despite their drawbacks, there are applications for novel, invasive, single use sensors, such that they complement clinical point-of-care applications by reducing 
demand for higher-order analytical imaging techniques. Continual sensors enable persistent monitoring and currently are most often used non-invasively for monitoring key physiological metrics such as heart rate, blood $\mathrm{O}_{2}$, or blood pressure [6]. Invasive, continual sensors are typically deployed alongside implanted medical devices for treating heart arrhythmias or diabetes; however, noninvasive monitoring techniques are preferred as they improve patient compliance while mitigating risk. From the implementations of biosensors outlined, the concept of precision health requires non-invasive continual monitoring to preemptively detect disease; however, there are many practical hurdles to overcome. Nonetheless, wearable devices are exploding onto the market as non-invasive continual sensors [7]. Driven by affordability and the proliferation of consumer electronics coupled with a growing public desire for health awareness, wearable devices, as non-invasive continuous sensors, are becoming increasingly popular for doctors and individuals to obtain continual medical-quality data. Leveraging existing technology, smart watches and cellphones can monitor a battery of key physiological metrics, including heart rate, activity levels, and blood pressure.

Molecular imaging research is often focused on probe development while electronic biosensing is often focused on achieving increased sensitivity of existing probes via development of novel sensing platforms. Combining validated molecular imaging probes with biosensing platforms could bridge the gap to provide both pre- and post-diagnosis monitoring. Clinical utility must be a key consideration, such that rapid, point-of-care, molecular sensors can be deployed as diagnostic tools in a non-laboratory setting as complementary or orthogonal methods to sophisticated multimodal molecular imaging techniques. Through multidisciplinary collaboration with clinicians to develop useful tools, the umbrella of clinically monitored physiological metrics can be broadened. In a complimentary manner, drug development can similarly be improved where biosensors can be used to screen the efficacy of therapeutics before clinical validation by molecular imaging. By integrating the precise, in vivo, data afforded by molecular imaging with the broad, continual data generated by multimodal molecular sensing for biomarker characterization, a positive feedback loop could be established. The process of characterizing known disease biomarkers could then identify new disease biomarkers and therapeutic targets as we fill in the gaps of the human model. Multimodal devices that can holistically monitor biomarkers of interest, paired with diagnostic molecular imaging techniques will enrich and modify, but not replace, how we prevent, treat, and monitor chronic diseases such as cancer, heart disease, and diabetes. While many technologies, including immunochromatographic assays, or behavior monitoring through optical scanners and semantic analysis will be necessary facets of the precision health umbrella [8], the aim of this review is to make the case that within the scope of electronic molecular sensors, many of the considerations in the implementation of molecular imaging are shared, and through multidisciplinary pairing of validated molecular imaging probes with electronic sensors we can alter the clinical paradigm and help realize precision health (Fig. 1).

\section{Electronic Sensing}

In the broadest sense, an electronic sensor is any device that recognizes changes to its environment by producing or altering an electrical signal that can be transduced into a useful result. Electronic sensors that can recognize biological elements can be termed biosensors and implemented
Fig. 1. Reimagining the clinical paradigm with paired molecular sensing and imaging. Through early sensor-based screenings and increasing the clinical monitoring umbrella, acute symptoms can be bypassed, and the effectiveness of treatments can be rapidly assessed. Molecular imaging in turn enables non-invasive diagnoses and improves surgical outcomes through increased surgical precision. Drug development and discovery can also be streamlined with both molecular sensing and imaging as more data, more immediately, is available.

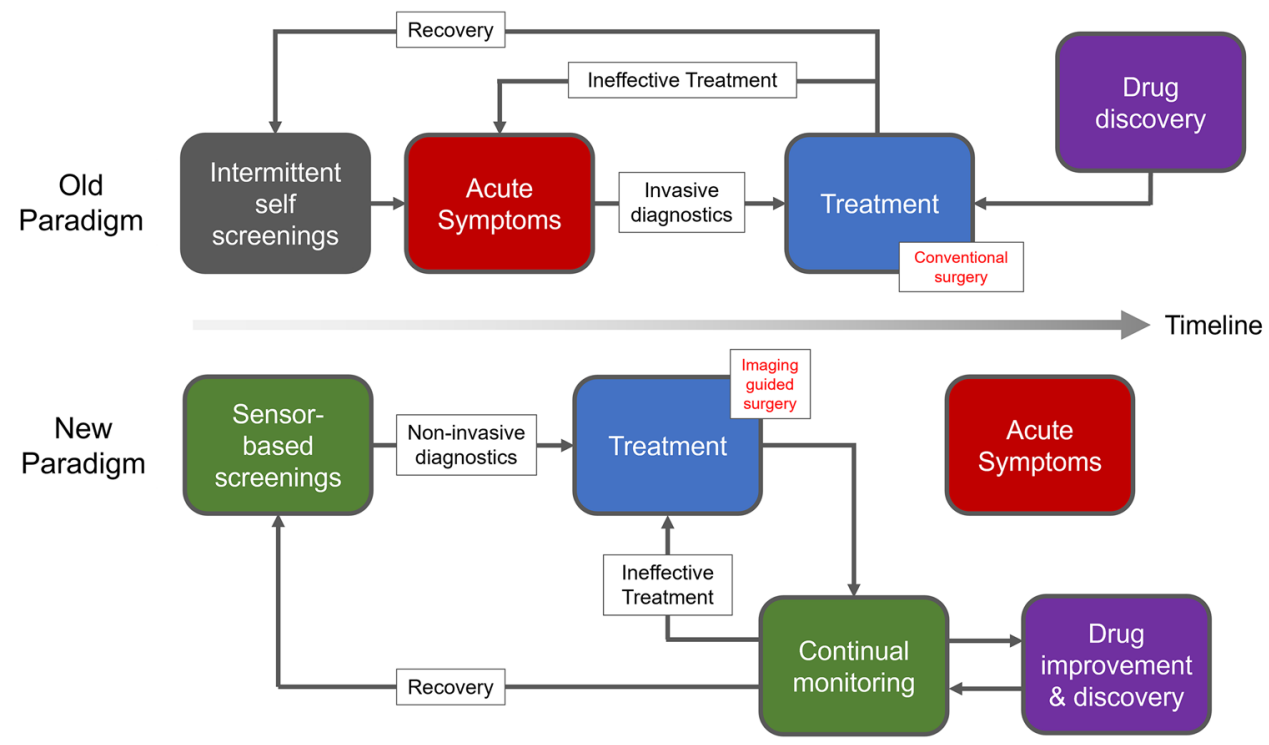


as molecular monitoring devices. For practical implementations, voltage $(V)$, current $(I)$, and resistance $(R)$ are recorded across a material and are dependent on each other by Ohm's law. While many electronic sensors are highly sensitive, the integration of molecular probes as a recognition element is often necessary to achieve useful selectivity. Analytes can interact directly with the sensing material or recognition element in gas or liquid phases, either through direct covalent bonds, indirect molecular interactions, or by altering the ionic potential of the solution, in each case, inducing a measurable change between electrodes (i.e., a change in $V$ ) [9]. Additional data points can be collected by modulating operating conditions which can be used to trigger redox reactions, alter resistance, or turn on semiconductors, providing real-time information about dynamic systems. Generally, for two-electrode systems, either $V, I$, or $R$, is held constant, and changes are measured in response to analytes, however, as variables dependent on each other, only having two electrodes limits the ability to perform multiparametric measurements and measurements across a range. By adding a third electrode, measurements can be conducted simultaneously between electrodes and, by maintaining a constant measurement at the intersecting electrode, multiparametric measurements can be taken and a range of potentials assayed. In a three-electrode transistor configuration, a dielectric insulating material between two of the electrodes can be used to generate a buildup of charge carriers at the interface of a semiconducting material across the other electrode junction. This charge buildup then drives charge transport through the semiconducting material, rendering the transistor in an "on" state. Forming the basis of computing and acting as self-amplifiers, transistorbased sensors can be readily integrated into circuits and fabricated on the nanoscale [10-12].

Organic semiconductors have been demonstrated for a variety of biosensing applications and are well suited to the task due to their mechanical adaptability. Every part of the three-electrode system (gate, source, and drain electrodes) can be modified with a broad range of materials and architectures to suit specific applications, permitting inherently biocompatible designs. For example, organic thin-film transistors (OTFTs) tend to be solid-state devices while electrolyte gated organic field effect transistors (EGOFETs) have an electrolyte solution as the gating material. The recognition of biological events can occur at the gate, dielectric, or semiconducting layers through functionalization with a probe molecule [12] such that a variety of altered electronic signals can be transduced into a useful readout. Many signal transduction processes elicit some combination of optical, electronic, and physical effects such that one method of signal transduction does not eliminate another. Thus, chemical immobilization of bioactive probes as sensing elements is a common strategy to functionalize the semiconducting channel of OTFTs with antibodies, oligonucleotides, proteins, and chemical probes. Combined with their low cost of manufacturing and implementation, electronic biosensors can readily be paired with the same or analogous sensing elements as molecular imaging probes, however pushing the boundaries of temporal testing resolution, ranging from high frequency to continual sampling of key biomarkers. Ultimately, the commonality of probe design and complementarity of testing regimens between molecular imaging and molecular sensing provides a means to populate the predictive human model that underlies precision health (Fig. 2).

\section{Clinical Opportunities for the Integration of Molecular Imaging with Molecular Sensing Devices}

Early detection of cancer and cardiovascular disease (CVD) are perhaps the most compelling arguments for the development of continuous point-of-care diagnostic sensors. The leading cause of death in many countries, these diseases exact a terrible social and economic toll [13]. Cancers are a highly complex group of diseases often only detected in the late stages, which limits therapeutic options and worsens prognoses. CVD is often caused by atherosclerosis resulting in coronary artery disease and ischemic strokes. Many forms of CVD have long asymptomatic phases during which inexpensive treatments or interventions can significantly improve health outcomes and limit the onset of adverse symptoms requiring hospitalization, if only the existence of this silent pathogenesis was identified. For cancers, once detected, a broad range of molecular imaging probes have been developed to assay the extent of disease dissemination and determine treatment options; however, they are limited to a hospital or laboratory setting. In both cases, early intervention significantly improves patient outcomes and reduces the societal cost burden. Thus, there is a critical need for devices for early screening, to facilitate early interventions, and to monitor the effects of treatment to provide early signals that the selected therapy is no longer effective [14].

As a current example, prostate examinations are performed manually and intermittently, with high-risk individuals tested by enzyme-linked immunosorbent assay (ELISA) for prostate-specific antigen (PSA), a biomarker elevated in the presence of some prostate cancers. The standard of care is to perform an ultrasound-guided biopsy; however, negative results do not guarantee a lack of prostate cancer. Increasingly, multiparametric MR imaging, leveraging antibody, aptamer, or small molecule probes, has been demonstrated clinically to assist in prostate cancer biopsies and serve as a diagnostic tool [15-18], with radiolabeled small molecule PET imaging used for postdiagnosis monitoring of severity and potential spread [19-21]. Though these molecular imaging techniques have 
Fig. 2. Electronic biosensor architypes and operation. Surface or electrode modifications with exposure to analytes induce altered electrical properties and with functionalization enabling selective responses. Increasing the number of electrodes increases design complexity; however, it also facilitates multiparametric measurements and complex operations such as electrochemical characterization and transistor operation. Molecular probes can be integrated into almost any components to accommodate a variety of sensor designs and implementations.

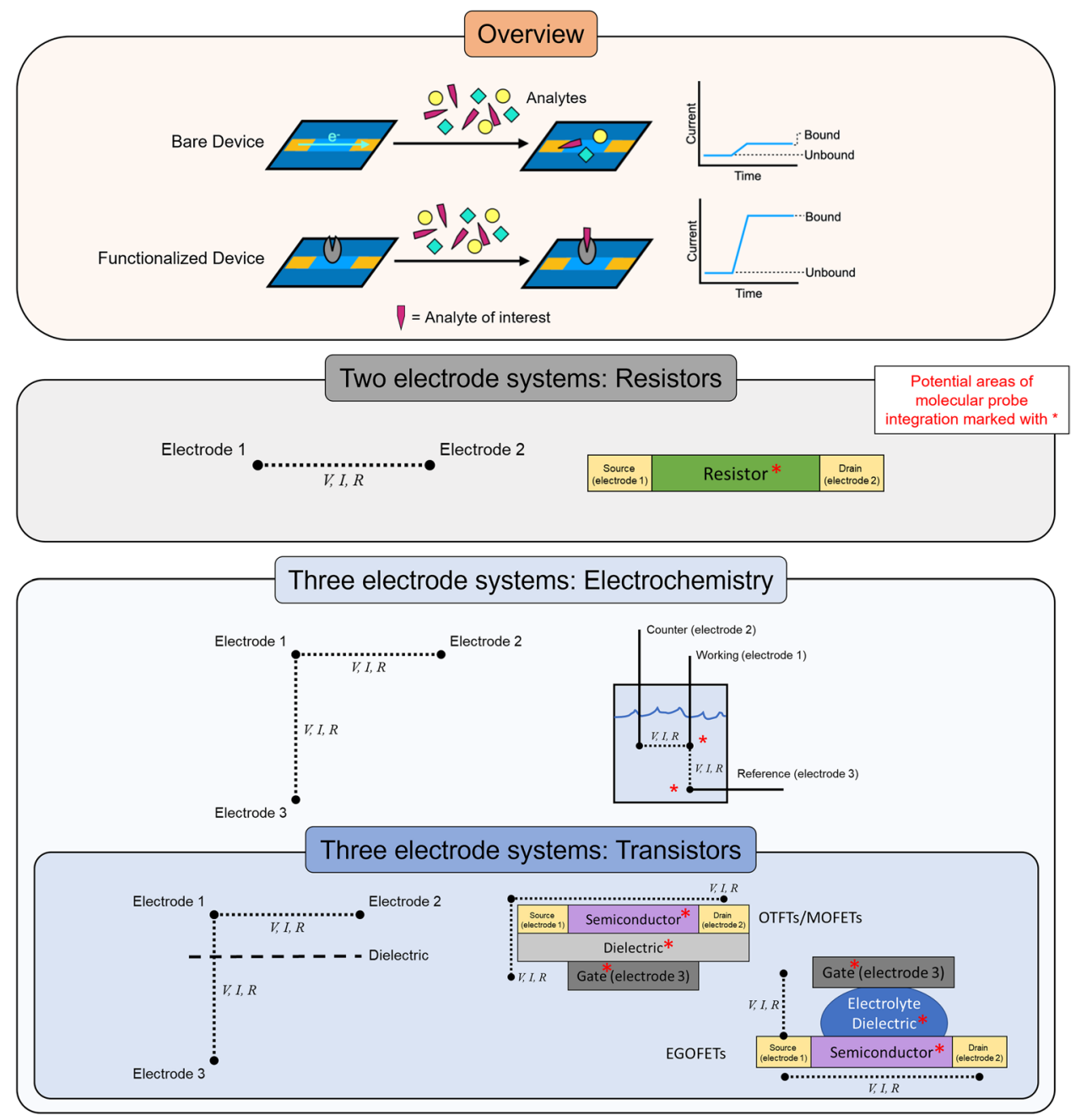

greatly improved the accuracy of clinical diagnoses and post-diagnosis monitoring, they are limited to the clinical environment, leaving gaps in post-diagnosis monitoring, and an un-met need for pre-diagnosis monitoring. To this end, a variety of electrochemical detection strategies have been developed utilizing functionalized antibodies to achieve clinically relevant detection limits [22-25]. Pyrolytic graphite, carbon nanotube forests, and gold nanoparticles are demonstrated as substrates for differential pulse voltammetry and amperometry-based electrochemical sensing in serum, potentially facilitating rapid detection of PSA in a clinical setting. Alternatively, electrochemiluminescent (ECL) labels have been combined with antibody-functionalized nanoparticles to amplify square wave voltammogram signal on carbon nanotube forest substrates [25]. Integrating sensors and imaging into the clinical pathway would permit continual screening for PSA or prostate-specific membrane antigen (PSMA), with molecular imaging serving to guide or replace biopsies for diagnosis should further intervention be necessary $[16-18,26]$. Minicircles, by delivering exogenously encoded reporter genes into cancer cells, offer an attractive method to endogenously produce both imaging and sensing probes, and could enable multimodal detection of prostate cancer aggressiveness [27]. Other predictive biomarkers (i.e., those that are cancer-predictive but not necessarily cancer-associated) have been identified and demonstrated in electrochemical devices including platelet factor 4 (PF-4), interleukin 6 (IL-6), interleukin 8 (IL-8), and carcinoembryonic antigen (CEA). Thus, during treatment, molecular sensors can be used to monitor the effects of therapies, providing rapid feedback to better direct clinical decisions [28-30]. Advances in molecular imaging and sensing can radically alter clinical workflows, with molecular sensors replacing intermittent invasive clinical monitoring and molecular imaging providing accurate, noninvasive, diagnoses.

As another example, glucose monitoring is critical to diabetes management and the role of glucose utilization as a pathogenic biomarker for of a variety of diseases, including kidney diseases, [31, 32] neurological conditions, $[33,34]$ and hepatic diseases [35] is well characterized. 
Table 1. A selection of probe types shared between imaging and sensing platforms to assay specific diseases.

\begin{tabular}{|c|c|c|c|c|}
\hline Target & Probe Type & Selected Biomarkers & Sensing Modalities & Imaging Modalities \\
\hline \multicolumn{5}{|l|}{ Cancer } \\
\hline \multirow[t]{2}{*}{ Pan-cancer biomarkers } & Antibody & $\begin{array}{l}\text { AFP, ferritin, CEA, hCG- } \beta \text {, CA } 15-3 \text {, CA } \\
125 \text {, CA } 19-9 \text {, carbonic anhydrase IX }\end{array}$ & Electrochemical $^{41,42}$, optoelectronic ${ }^{25}$ & $\mathrm{PET}^{43,44}, \mathrm{MRI}^{44}$, Optical $^{44}$ \\
\hline & Aptamer & AFP, CEA, miRNA, CD30 & Optoelectronic $^{45}$, Electrochemical $^{46}$ & $\mathrm{PET}^{47}$ \\
\hline \multirow[t]{2}{*}{ Breast cancer } & Antibody & c-erbB-2, CEA, MUC1, TAG-72 & OTFT $^{48}$ & $\mathrm{PET}^{49,50}$ \\
\hline & Aptamer & SK-BR-3, MCF-7 & Nanopore $^{51}$ & Optical $^{52}$ \\
\hline \multirow[t]{3}{*}{ Lung cancer } & Antibody & $\begin{array}{l}\text { ANXA2, CKAP4, ENO1, VEGF, NR-LU- } \\
10\end{array}$ & Impedance ${ }^{53,54}$ & PET $^{55,56}$, Optical $^{57}$ \\
\hline & Protein & CEA, NSE, KLKB1 & Electrochemical $^{54}$ & $\mathrm{PET}^{55}$ \\
\hline & Small Molecule & VEGF165, EGFR, CK19 & Impedance $^{58}$ & $\mathrm{PET} / \mathrm{CT}^{59}$ \\
\hline \multirow[t]{4}{*}{ PSA } & Antibody & PSA, PSMA & Electrochemical $^{23,60,61}$, OTFT $^{62}$ & $\mathrm{MRI}^{63}, \mathrm{PET}^{64}$ \\
\hline & Aptamer & PSA & Nanowire $^{65,66}$, optoelectronic $^{45}$ & $\mathrm{MRI}^{67}$ \\
\hline & Protein & PSA & Electrode $^{68}$ & \\
\hline & Small molecule & PSA, PSMA & & $\mathrm{PET}^{21,69}, \mathrm{MRI}^{19}$ \\
\hline \multicolumn{5}{|l|}{ Coronary artery disease } \\
\hline & Antibody & CRP, TNT- $\alpha$, IL- 6 , inflammatory cells & Electrochemical $^{70}$, OTFT $^{71}$ & $\mathrm{PET}^{72}$ \\
\hline & Aptamer & Troponin-T, thrombin, IL-6 & Electrochemical $^{73,74,42}$ & $\mathrm{US}^{75}$ \\
\hline & Small molecule & Lipoproteins, EP-2104R & Electrochemical $^{76,70}$ & $\mathrm{PET}^{77,72,78}, \mathrm{MRI}^{72,78}$ \\
\hline \multicolumn{5}{|l|}{ Neurological } \\
\hline \multirow[t]{5}{*}{ Alzheimer's disease } & Antibody & $\mathrm{A} \beta_{42}, \mathrm{~A} \beta_{40}, \mathrm{p}-\operatorname{tau}_{181}$ & Impedance $^{79,80}$, CNT Resistor ${ }^{81}$ & $\mathrm{PET}^{82-85}$ \\
\hline & Aptamer & Thrombin, adenosine, $\mathrm{A} \beta \mathrm{O}, \mathrm{A} \beta_{40}$ & Nanowire $^{86}$ & Optical $^{87}$ \\
\hline & Protein & $\mathrm{A} \beta \mathrm{O}$ & Electrode $^{88}$ & \\
\hline & Small molecule & $\mathrm{A} \beta, \mathrm{p}-\mathrm{tau}_{217}$ & & MRI $^{89}$, PET $^{90-92}$ \\
\hline & Quantum Dot & QDA $\beta$ & Optoelectronic $^{93}$ & \\
\hline \multirow[t]{4}{*}{ Dopamine dysregulation } & Antibody & -- & Electrochemical $^{94}$ & \\
\hline & Aptamer & -- & OTFT $^{95}$, electrochemical ${ }^{94}$ & Optical $^{96}$ \\
\hline & Enzyme & -- & Biosensors $^{94}$ & \\
\hline & Small molecule & -- & & $\mathrm{PET}^{97,98}$ \\
\hline
\end{tabular}

Through the facile immobilization and implementation of glucose oxidase (GOx) for electrochemical sensing, a range of architectures for electrochemical glucose biosensing have been implemented, including transdermal glucose monitoring. Designed as an alternative to finger prick blood draws, transdermal sensors can noninvasively measure blood glucose concentration in real time, aiding in diabetes management. However, incorporated into CVD therapies, transdermal glucose monitoring facilitates better glycemic control during and post-surgery which has been recently shown to improve patient outcomes [36]. For both cancer and CVD, aided by well-developed chemistry permitting the radiolabeling of glucose for PET imaging, imaging-guided surgery has similarly proved to be an invaluable tool toward improving patient outcomes [4, 37-40]. Thus, for surgical applications, molecular imaging and sensing could work in tandem, guiding surgical procedures, minimizing invasiveness, and improving recovery through more precise health monitoring. In addition to the examples described above, a myriad of imaging techniques and sensing platforms have been developed to target a range of diseases (Table 1).

\section{Shared Probe Design Considerations}

Molecular imaging probes are agents used to visualize biochemical processes by providing a signal that can be detected by the camera for the imaging modality of choice. Thus, a signaling agent or beacon specific to the imaging modality is often linked to the targeting moiety which interacts with the biomarker of interest. Similarly, probes for electronic biosensing require a targeting moiety in addition to a handle or linker moiety through which the probe can be conjugated to the surface of the device, allowing the conversion of analyte-probe interaction into an electronic signal and facilitating its transduction to the device. As a result, probe design for molecular imaging shares many of the same considerations as probe design for molecular sensing devices. Molecular imaging and sensing can be complimentary techniques from both a clinical aspect and a design aspect. By targeting the same biomarkers or by using the same recognition agents, advances in either can often be repurposed to potentially innovate in the orthogonal technique. Furthermore, clinically, where molecular imaging can provide focused analysis of disease state in vivo, molecular sensing can complement imaging techniques, 
providing continuous analysis of disease progression at home or clinically at the bedside (Fig. 3).

\section{Antibodies}

Functionalized antibodies have become popular targeting moieties due to their well-defined structure, specificity, high affinity, and serum stability, positioning them well for use as targeting agents for molecular imaging probes and electronic biosensors. Antibodies generally consist of a constant region, termed heavy chain, a light chain, and a variable region with the antigen-binding site. Through a complex, naturally occurring, V(D)J B-cell recombination process, a staggering diversity of receptors are possible, [99] enabling antibody-based targeting of a broad range of biomarker targets. The constant regions, while not conferring antigen-binding specificity, are essential for mediating the resulting immune response and cytotoxicity. Thus, depending on the organism used to produce the antibodies, they can elicit an unwanted immune response in humans; however, developments in antibody engineering and antibody fragments have addressed some of these challenges without compromising functionality [100]. For both imaging and sensing, site-specific labelling of amino acids guides conjugation strategy, which is a key challenge in employing antibodies as targeting agents and a rapidly expanding area of research [101-103]. With smart antibody design and shared click chemistry, a recombinant antibody ( $\mathrm{rAb}$ ) could be clicked to both a sensing platform and an imaging agent, providing instant sensing and imaging of a specific biomarker. The affinity and specificity of the antigen-binding region would be maintained, facilitating orthogonal imaging and sensing of a specific antigen provided appropriate antigen bioavailability. Enabling imaging of intracellular targets, antibody fragments can facilitate click chemistry while also limiting background signal and non-specific accumulation; however, their practical use is still an on-going challenge $[44,104,105]$.

As an example of the potential flexibility of antibodybased detection agents, functionalized hafnium oxide $\left(\mathrm{HfO}_{2}\right)$ substrates were demonstrated for hybrid field effect transistorimmunofluorescent detection of human interleukin (IL)-10, [106] a biomarker associated with inflammation and adverse cardiovascular events [107]. Detecting IL-10 electronically by conjugating the antibody to the $\mathrm{HfO}_{2}$ surface, rather than optically, afforded a 30 -fold increase in device sensitivity versus fluorescence detection [108]. In both cases, a harsh silane treatment was used to functionalize the surface with available aldehydes enabling mild covalent antibody coupling. While a variety of alternative techniques exist for antibody-device surface coupling (e.g., thiol, glycan, non-covalent immobilization, or affinity-based immobilization), a key aspect to consider however with any site non-specific method is a reduction in the availability of antigen-binding sites due to random immobilization. Antibody fragments obtained from rAbs can again be used to limit this effect with the additional advantage of enabling a higher density of antigen-binding sites on the substrate surface.

Upstream in the development process, electronic biosensors with immobilized rAb targets on their substrate surface could find a role for $\mathrm{rAb}$ evaluation, providing additional screening data on their specificity and affinity. Electronic sensors can then assist antibody imaging both by facilitating the selection of lead rAbs and providing a complimentary assay technique for disease specific antigens. With their advantages for both imaging and sensing, antibody fragments are well

\section{Probe Design Considerations}

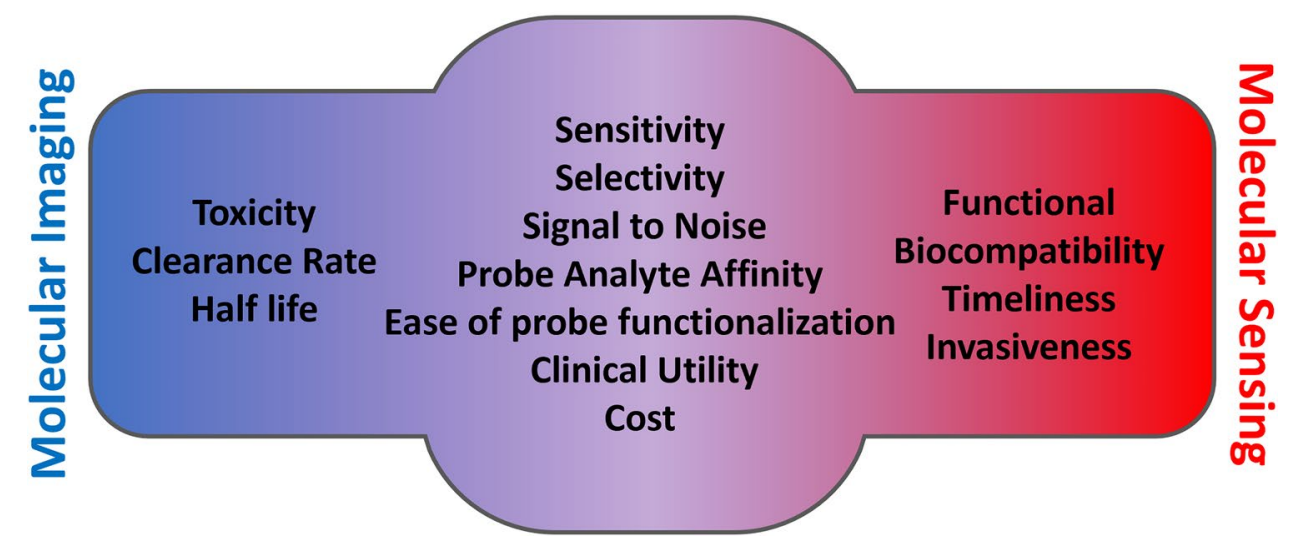

Fig. 3. Shared design considerations of molecular imaging and molecular sensing probes. Probes for molecular imaging and molecular sensing share many of the same considerations as they pertain to analyte interaction and signal transduction and thus probes which work for one technique may be applicable to another. Molecular imaging and sensing instead slightly differ in relation to the practical aspects of each technique. Where imaging agents are typically injected into the subject, and thus toxicity, the rate of clearance, and half-life are major concerns, sensors are often outside the subject, interacting only partially with biofluids, and thus necessitate functional biocompatibility, timeliness of response, and minimizing the sampling invasiveness. 
positioned to potentially serve to generate paired molecular imaging agents and molecular monitoring devices [109-111].

\section{Oligonucleotides}

Oligonucleotides, either as immobilized strands of DNA or RNA, have been demonstrated as recognition elements in a variety of electrical biosensors [112] and imaging probes. Functionalization is facilitated by chemically modifying either the $3^{\prime}$ ' or 5' ends of oligonucleotides with well-established chemistry. Oligonucleotides can be readily labelled, enabling application to a broad range of imaging modalities while producing few changes in the behavior of the molecules. Oligonucleotides are produced with great facility and at lower costs in comparison with enzyme- or antibody-based methods for analyte recognition. With limited immunogenicity or off-target toxicity, oligonucleotide-based probes offer an attractive alternative to other probe systems. Uniquely, oligonucleotides provide rapid and highly specific hybridization to complementary strands amenable to the growing interest in cell-free RNA and DNA as biomarkers of disease. Oligonucleotide functionalization is not without challenges as the probes can have short half lives in vivo and must have a well-defined orientation that is accessible for hybridization.

Independently from hybridization (i.e., base-base recognition), but rather as unique oligonucleotide folding conformations, aptamers can provide highly specific recognition sites enabling their use as molecular imaging and sensing probes. Selectively screened from a complex library in vitro for high affinity and specificity, aptamers can bind with a broad range of biomolecular targets and have been demonstrated in OTFT sensors to detect a range of small molecules, peptides, and proteins $[11,95,113,114]$. Aptamers can be fabricated costeffectively and afford enhanced stability in response to $\mathrm{pH}$, temperatures, and solutions of high ionic strength with in vivo stability in the range of hours [115]. This combination of ease of production, consistent structure, and stability positions aptamers well as a flexible imaging and sensing recognition agent [73, 116-118].

\section{Small Molecules}

Small molecule probes have found broad applications in a variety of imaging modalities through incorporation of an imaging beacon, and they are also readily immobilized on electronic devices to afford analyte sensing. Advances in PET and SPECT imaging and radiochemistry have enabled incorporation of ${ }^{11} \mathrm{C}$ and ${ }^{18} \mathrm{~F}$ radionuclei, among other radioisotopes, into small molecule structures enabling the study of complex metabolic pathways with the advantage of limiting disruptions in molecular behavior. As targeting agents for sensing, small molecules that form covalent bonds or undergo redox reactions with the analyte at an electrode interface is often all that is required to produce a measurable sensing response [119-122] in some capacities, enabling single molecule sensing [123]. However, while sensitivity can be as high as single molecule detection, selectivity and specificity is a core challenge to broader sensor use. As a result, significant effort has been directed at developing a variety of sensing platforms to target small molecules, often by interpreting specific analyte-probe interaction-induced conformational changes as electrical signals [124-126]. Electronic noses, wherein an array of slightly varied sensors facilitates deconvolution of non-specific molecular events, can be used to improve selectivity. We demonstrated the integration of a colorimetric cannabinoid probe as sensitizing agent with OTFT-based sensors and then through material selection were able to selectively determine cannabinoid ratios from a complex solution [127]. Other colorimetric probes have been integrated into optoelectronic noses [128], most commonly for airborne smallmolecule targets such as aldehydes and ketones.

As sensing targets, aldehyde and ketone dysregulation and their associated enzymes have been used as an index of oxidative stress and inflammation and therefore can be used as biomarkers for a variety of disease states [129-131]. Aldehydes represent a promising target for molecular sensing as they have high bioavailability in biological fluids that can be sampled relatively non-invasively. Additionally, specific environmental aldehydes can cause negative health effects, affording their use as indicators of pollution and spoiled food. Acetaldehyde and ketone breath sensors, using metal-oxide semiconductor field-effect transistor (MOSFET) devices as the electronic transducer, are widely available as breathalyzers to analyze alcohol consumption and to monitor ketogenic diets, respectively [132]. Imaging aldehydic load could provide a more holistic approach toward understanding disease pathogenesis, and non-invasively measuring aldehyde concentration in available biofluids has been demonstrated as a useful diagnostic metric. Shuhendler and coworkers have developed a series of aldehyde probes, leveraging hydrazone formation to provide rapid aldehyde complexation without a catalyst [133]. While their clinical use and utility is still limited, volatile organic compound (VOC) breath or environmental sensors can fit into the umbrella of precision health by providing additional complimentary data to small molecule imaging studies.

\section{Enzymes and Reporter Genes}

Since the demonstration of immobilized glucose oxidase (GOx) in 1962 as a glucose sensor, a popular technique for electrochemical biosensing is monitoring electroactive enzymatic products via oxidation or reduction reactions. Through the generation of oxygen by GOx, the activity, and thus the concentration of glucose, can be monitored electronically in real time. Glucose test strips expanded on this principle, miniaturizing and commercializing the technology in the late 1980s. Recently, transdermal, continuous glucose monitoring has been demonstrated for diabetes and surgical monitoring, improving individuals' quality of life and surgical outcomes, respectively [36]. 
Reporter genes are a key part of molecular imaging as efficient tools to monitor the efficacy of gene delivery, gene expression, and facilitating imaging of challenging molecular targets. In this way, enzymatic activity can be imaged in vivo. As an example, $\left[{ }^{18} \mathrm{~F}\right]$-trimethoprim ( $\left.\left[{ }^{18} \mathrm{~F}\right]-\mathrm{TMP}\right)$ was used to image the expression of Escherichia coli dihyrofolate reductase enzyme (eDHFR) enabling the trafficking of human chimeric antigen receptor $\mathrm{T}$ cells to be followed in mice [134]. TMP is a popular antibiotic used alongside sulfamethoxazole (SMX) to treat urinary tract and ear infections. Electrochemical sensors have been developed and demonstrated to simultaneously detect SMX and TMP in urine [135] potentially facilitating multimodal, and continuous monitoring of TMP pharmacodynamics. Minicircle plasmids have demonstrated tumor activatable paired sensing and imaging reporter gene, producing easily assayed biomarkers for sensing in combination with imaging markers, and demonstrating the potential of pairing molecular imaging and sensing from the design stage [27, 136, 137].

Sensor-functionalized enzymes can play an important role in drug development through real-time assaying of enzymatic activity, corroborating in vivo imaging studies. Xanthine oxidase (XO) catalyzes the oxidation of hypoxanthine (HYP) to xanthine (XAN), which is then further oxidized to uric acid as the final degradation product in the human purine catabolic pathway. Overproduction of these degradation products can lead to xanthinuria, hyperuricemia, high blood pressure, gout, and renal failure. Allopurinol (1H-pyrazolo[3,4-d] pyrimidin4-ol) is a XO inhibitor in clinical use to treat gout and improve long-term outcomes following ischemic strokes [119, 138]; however, as established through molecular imaging studies, there can be a variety of strong side effects [139, 140]. Through simple and sensitive amperometric biosensors with surface functionalized XO, the chemical mechanism of allopurinol inhibition was determined [141]. Building on the premise, allopurinol alternatives for XO inhibition were able to be rapidly tested ex vivo [142]. Thus, molecular sensors can assist with drug development before molecular imaging can be used to validate clinical effectiveness. With the ability to detect the downstream products of XO activity, these sensors could also fill a role in disease monitoring.

\section{Monitoring the Human Engine- Blood, Sweat, and Tears}

Molecular imaging is focused on finding specific biomolecular targets in living systems and thus key considerations of imaging probe design are focused on delivery, specificity, and clearance. Analyte availability is a secondary concern in so far that the targeted analyte is usually found in the highest concentrations at the site of interest (e.g., site of disease initiation). For molecular sensing, particularly when developed in combination with imaging probes, analyte availability and its biodistribution are the primary concerns such that the concentrations of analytes available in sampled biological fluids can be detected, and that the analyte levels evaluated from a biological sample are reflective of the disease state. To this end, the routes by which biomarkers are shed, processed, and excreted, and the invasiveness by which the biofluids in that process can be sampled, is a key consideration of molecular sensor design. Compartmentalization, both in respect to cells and larger bodily systems is an additional concern. To draw on the analogy of an engine, an issue with the engine oil is unlikely to be detected in the wiper fluid; thus, analyte bioavailability is particularly relevant in the context of pairing molecular imaging probes with sensors. Whereas molecular imaging probes can target specific processes or intracellular analytes, molecular sensors are exclusively limited, for now, to extracellularly available analytes. Finally, functional biocompatibility is a necessary consideration when designing sensors, specifically, designing for electronics for operation in aqueous, and often ionic environments (Fig. 4).

\section{Breath}

Thousands of volatile organic compounds (VOCs) have been identified in human breath, providing an array of biomarkers that can potentially be targeted for analysis [154]. Alcohols, ketones, and aldehydes are popular VOC-based biomarkers for breath analysis as they can be detected directly by an electrode and somewhat selectively without the need of an intervening recognition element. However, a variety of human factors may influence breath sampling, including breathing route, exhalation rate, airway pressure, and posture [155]. Additionally, breath is not a homogenous mixture of gases [156]. Instead, biomarkers stemming from the oral cavity will be in higher concentrations in early breath (such as $\mathrm{H}_{2} \mathrm{~S}$ for halitosis [157]), while endtidal breath will have higher concentrations of blood-borne biomarkers (such as acetone [158]). Thus, flow restrictors are often necessary to achieve a consistent and prolonged exhalation for meaningful and reproducible analysis. Nonetheless, metal-oxide chemiresistors, borrowing ideas from industrial gas monitoring, have been sensitized to detect biologically relevant levels of VOCs for monitoring metabolic disorders. To simultaneously analyze a broad range of aldehydes, optoelectronic noses have been developed, leveraging an array of dyes to electro-optically determine specific aldehydes and ketones [128]. Probe molecules incorporating a hydrazine binding moiety have been demonstrated for molecular imaging of aldehydes, converting an aldehyde binding event into fluorescent, MRI, or PETdetectable signal [135, 159-161]. Further sensitization can be achieved through device design, where hollow structure nanomaterials [162], carbon nanotube structures [163], adsorption traps [164], or buffering [165] are tailored to improve analyte delivery to the sensor. Breath sensing is a promising option to detect slowly progressing diseases with few early indicators [166], such as cancer [167], diabetes [168], or renal dysfunctions [169]. 


\section{Monitoring the Human Engine}

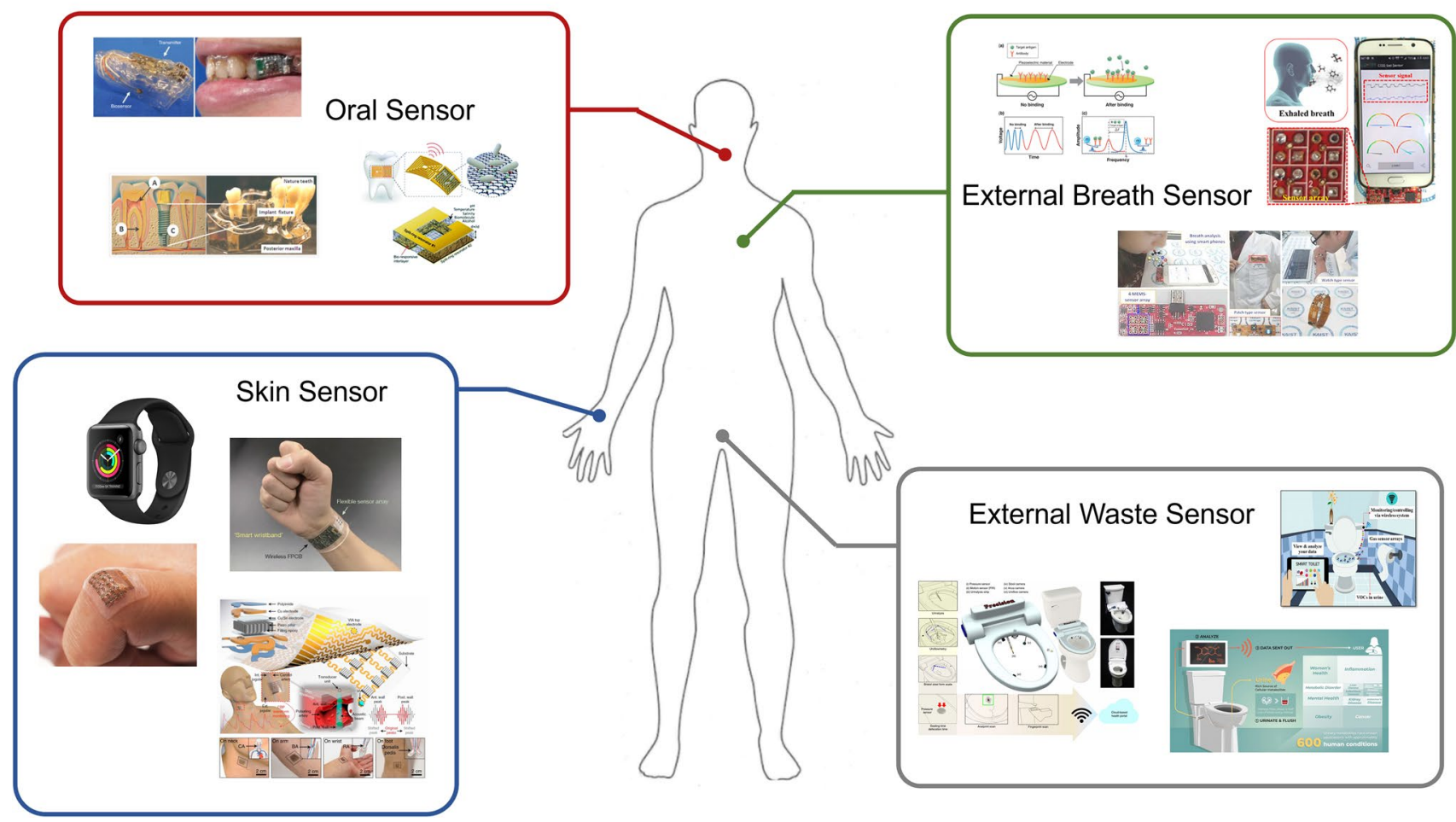

Fig. 4. Monitoring the human engine. Examples of recent works to incorporating non-invasive technologies to molecularly monitor a battery of health metrics. While physiological monitoring has become ubiquitous there is a current need for monitoring devices to provide clinically actionable diagnostic data. Complex biomarker monitoring is possible with molecular imaging but still limited in capacity with molecular sensing. (a) Schematic image of a glucose biosensor with mouthguard support and oral implementation ( adapted from ref.146). (b) Proposed intra-oral dental implant system with an integrated three-electrode electrochemical biosensor (from ref.147). (c) Biotransferrable graphene wireless nanosensor (adapted from ref.148). (d) Apple Watch Series 3 with integrated heart rate and blood pressure sensors. (e) A wearable fully integrated sensor array on a subjects wrist for in-situ perspiration analysis (adapted from ref.149). (f, g) Stretchable ultrasonic devices and design schematic for monitoring the central blood pressure waveform (adapted from ref.150). (h) Function of a immuno-piezoelectric biosensor (adapted from ref.151). (i) Electrochemiresistive breath sensing with apoferritin encapsulated nanoparticles (from ref.152) (j) Real-time breath analysis via portable functionalized electrochemical sensing platforms (from ref.153). (k) A mountable toilet system for personalized health monitoring (from ref.154). (I) Real-time health monitoring through urine metabolomics (from ref.155). (m) Urine odor detection by electronic nose for smart toilet applications (from ref.156).

\section{Saliva}

Saliva is being increasingly recognized as a useful diagnostic fluid for biomarker analysis as it can be easily and noninvasively sampled [170]. Human oral fluid is mainly composed of saliva produced by three pairs of major salivary glands and a large number of minor salivary glands. Each salivary gland secretes a characteristic type of saliva with variations observed in the concentrations of salts, ions, and total proteins [171-173]. Additionally, saliva contains crevicular fluids, oropharyngeal mucosae, blood-derived compounds, and food debris, with the average healthy adult producing $500-1500 \mathrm{~mL}$ per day [170]. For sensing purposes, saliva is almost universally collected and analyzed whole, as separation or precise collection is often not practicable. Salivary glucose, lactate, phosphate, alpha-amylase, hormones, and antibodies have been demonstrated as sensing targets [170,
174, 175]. Miniaturized GOx sensors have been developed for noninvasive sampling of salivary glucose as well as crevicular fluid glucose, correlating these measurements to blood glucose concentrations with the aim of noninvasive monitoring for diabetes management $[176,177]$. Using the same principle as glucose measurements, salivary lactate can also be measured by electrochemical measurement of $\mathrm{H}_{2} \mathrm{O}_{2}$ generation from immobilized lactate oxidase. Elevated levels of salivary lactate can be indicative of lactic acidosis, a biomarker that can signal an impending heart attack [178, 179]. Several salivary lactate electronic biosensors have been reported, as electrochemical sensors [179] as well as miniaturized cavity-type sensors meant to be worn in the mouth, reporting salivary lactate levels in real-time wirelessly [180]. Salivary lactate concentrations also have consequences on sports performance, with a three-electrode monitoring system printed on the inside of a mouth guard demonstrating a 
sensitive and stable response [181]. Electrochemical immobilized enzyme sensors have also been reported for salivary phosphate [175], a cardiovascular calcification biomarker [182], and salivary alpha-amylase [174, 183], a stress biomarker [184]. Many blood serum hormones which can also function as biomarkers have been detected in saliva, suggesting promising noninvasive alternatives to blood serum testing. Electrochemical immunosensors have been demonstrated for monitoring adrenal biomarkers (cortisol [185, 186], testosterone [187]), cancer biomarkers (IL-8 [188], IL-1 [189], CD-59 [190]), and other disease biomarkers such as cytokine interferon [191], and SARS-CoV-2 [192]. With the popularity of oral implants for orthodontic purposes, non-invasive oral implants or sensors integrated into dentures have been demonstrated to provide real-time passive health monitoring.

\section{Sweat}

The average adult human produces $500-700 \mathrm{~mL}$ of sweat under most climate conditions per day [193]. Easily accessible, sweat can provide a range of physiological data including electrolyte levels, and metabolites [194]. It can be sampled continually and noninvasively with a skin patch, often preferable to a dental implant, and significantly preferable to blood collection. Gao et al. (2016) demonstrated a flexible integrated sensing array able to continually monitor glucose, lactate, sodium, potassium, and temperature in sweat, leveraging existing GOx and LOx enzyme-based electrochemical sensors in combination with $\mathrm{NaCl}$ and $\mathrm{KCl}$ ion sensitive electrodes. Temperature measurements were used to calibrate the activity of the other sensors and were able to continually monitor sweat produced during vigorous exercise. Sebum, the oily substance produced by sebaceous glands, contains non-invasively accessible lipids which have been demonstrated as neurodegenerative disease biomarkers [195, 196]. While a plethora of physiological information is present in sweat [146], there have been limited validated correlations of compartmental analyte availability and the relationships to physiological levels [197]. Recent advances in wearable electronics have begun to address these shortcomings but there is a need for paired imaging studies to validate the clinical value of sweat-borne biomarkers [146, 198]. Thus, the continuous physiological monitoring umbrella could be expanded from heart rate, blood pressure, and blood $\mathrm{O}_{2}$ to include blood glucose, lactate, and ions to improve overall health monitoring.

\section{Urine and Feces}

Monitoring of human waste has gained increased attention as a complementary alternative to blood plasma sampling. Blood plasma is controlled through tight homeostatic mechanisms, with deviations often signaling a major issue, and as a result the biomarkers found within are often short lived in vivo, limiting their use for early detection and continual monitoring. Waste, specifically urine, comprises objects cleared of the blood homeostasis mechanism, and can potentially better reflect in vivo changes. Additionally, the urine proteome is relatively low, facilitating analysis and validation of potential biomarkers. Broad, multiplexed urinalysis and the implementation of machine learning algorithms have found promising biomarkers to detect the early onset of a variety of diseases and infections [199-201], potentially facilitating early interventions of colorectal and prostate cancers. By leveraging the renal clearance of many therapeutics, including molecular imaging agents, additional information toward their effectiveness can be obtained. Acute kidney injury (AKI) is a common and serious complication following significant medical events and its early detection and treatment can reduce mortality and improve recovery following surgery [202-204]. It has been suggested that urine could be passively monitored from within the home in a similar fashion to CO monitoring, where out of bounds biomarker levels could trigger an alarm and further investigation. Changes to an individual's hydration and activity levels can act as a confounding factor and fouling can be an issue. "Smart toilets," leveraging a multi-sensor design, offer an interesting option for passive, noninvasive home health monitoring [81, 205]. By combining an array of existing waste monitoring sensors with machine learning, significant health data can be collected in a facile non-intrusive way. Integration of sensors for a specific purpose, such as a guaiac fecal occult blood test (gFOBT), microbiome, or biochemical analyses could enable early disease detection and long-term monitoring. Fecal tracking has been deployed extensively throughout the COVID-19 pandemic and largescale waste monitoring can provide significant state level public health data for population modeling [206]. Thus, "smart toilets" are expected to have significant impacts on health monitoring research and public health planning.

\section{Blood}

Blood plasma is considered to be the main target of research for biomarker analysis with proteomics and lipidomics identifying tens of thousands of molecular species and thousands of potential biomarkers for a wide variety of diseases. Repeat, comprehensive analysis of an individual's blood metabolome is impractical for clinical applications, necessitating targeted testing. Additionally, blood sampling requires invasive procedures and is available in limited quantities. Finger pricks are tolerable however analyte concentrations will be very low. Large ( $\mathrm{mL}$ volume) blood draws are currently used for "blood work" as a stopgap. Whole blood samples often further require separation steps, removing platelets and red blood cells to yield either plasma (with clotting factors) or serum (without clotting factors). It is preferential to avoid requiring a blood draw; however, alternative methods of biomarker acquisition may be impossible or impractical. Continuous blood and interstitial fluid monitoring has been demonstrated with implantable devices, [207] with advances in biocompatible implant materials bringing such devices closer to routine clinical use. With implantable devices currently being mostly experimental, external molecular sensors could be deployed 
Fig. 5. Advantages and disadvantages of commonly assayed biofluids. Biomarker availability is plotted against invasiveness. Ideally, molecular biosensors are as non-invasive as possible; however, biomarker availability for molecular sensing, and thus sampling considerations, is directly related to the routes by which it's shed, processed, and excreted. Where molecular imaging considers probe delivery to compartments, with probe metabolism and excretion being tied to clearance rate, molecular sensing must instead consider biomarker metabolism and excretion, the relationship to biomarker availability, and the invasiveness by which the biofluid can be assayed.

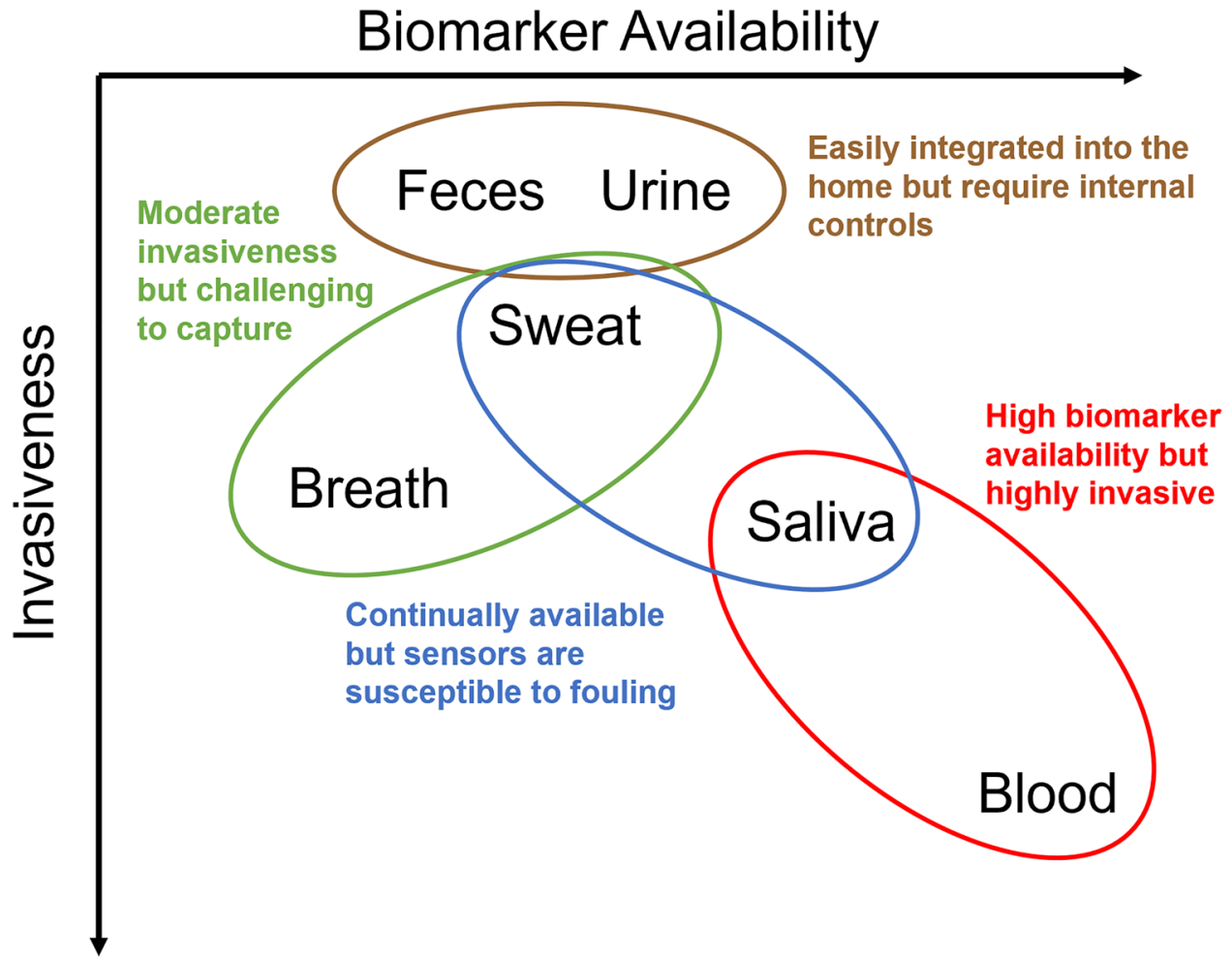

alongside other medical implants, such as pacemakers, or insulin pumps. Limiting the risks of infection and inflammation post-implant are still significant outstanding issues toward integration, in addition to challenges with wireless communication and regulations. Many implantable devices are classified as Class III devices by the FDA, necessitating a lengthy and stringent testing process to ensure reliability and safety. Implementation and integration of implantable devices into the clinical paradigm will require multidisciplinary collaboration to overcome the hurdles presented (Fig. 5).

\section{Wearables}

With the proliferation and miniaturization of cell phones and wearable devices, smart watches have become increasingly popular in the consumer market. Coupled with a growing societal focus on health, there is demand for additional personal health monitoring. However, many wearable devices available to consumers are only incorporating technology that has been available for decades. There have been marked improvements in miniaturization, ergonomics, and implementation, but the majority of the biosensors discussed herein have yet to be implemented for consumer, or even clinical use. As sensors, the usual considerations of sensitivity, selectivity, and feasibility apply, however, as long term, external, continuous sensors operating outside of a clinical environment they must also overcome environmental factors without fouling. Additionally, a bottleneck for future sensor implementation, particularly implantable sensors, is power supply. While modern batteries can last years, this limits the power draw of implantable sensors and thus their complexity. Both self-powered sensors and wireless charged sensors are in development to offer potential solutions to this challenge. Non-invasive sensors are preferred as they can be implemented with less risk of injury or side effects, and ultimately improve patient compliance.

\section{Conclusions}

Advances in molecular imaging have been a major driver toward changing clinical methods to better accommodate individual differences, personalizing medicine. This has prompted a recent push toward achieving precision health, shifting the clinical paradigm from reactive medicine to preventative medicine. To this end, molecular imaging is still generally limited to the clinical setting. By pairing molecular imaging with molecular sensing, design considerations and analyte recognition performance gleaned from imaging probes for validated biomarkers can be applied to the development of molecular monitoring devices and moved out of the clinical setting and into the home. Though still limited in consumer available implementations, improvements in wearable health technology, its increased use in our daily lives, and further integration with clinician available electronic health records will dramatically change how we diagnose, monitor, and treat disease. With earlier disease diagnoses, patient outcomes are improved, while societal and economic health burdens are lessened. Within clinical settings, 
molecular imaging integrated with molecular sensing could broaden our understanding of disease progression, generate data necessary to complete the human model, and create positive feedback loops toward identifying earlier biomarkers and better therapeutics.

Funding This work was supported by NSERC Discovery grant RGPIN 2015-05796 (A.J.S.), and 2020-04079 (B.H.L.), the Canada Research Chairs Program 950-230754 (A.J.S.), and 950-230724 (B.H.L.).

\section{Declarations}

Conflicts of Interest The authors declare they have no conflict of interest.

\section{References}

1. Gambhir, S. S., Ge, T. J., Vermesh, O. \& Spitler, R. Toward achieving precision health. Sci. Transl. Med. 10, (2018).

2. Gambhir, S. S., Ge, T. J., Vermesh, O., Spitler, R. \& Gold, G. E. Continuous health monitoring: An opportunity for precision health. Sci. Transl. Med. 13, (2021).

3. Collins FS, Varmus H (2015) A New Initiative on Precision Medicine. N. Engl. J. Med. 372:793-795

4. Wang C et al (2019) Advanced Nanotechnology Leading the Way to Multimodal Imaging-Guided Precision Surgical Therapy. Adv. Mater. 31:1904329

5. Terris M (1975) Evolution of Public Health and Preventive Medicine in the United States. Am. J. Public Health 65:161-169

6. Mendelson Y (1992) Pulse Oximetry: Theory and Applications for Noninvasive Monitoring. Clin. Chem. 38:1601-1607

7. Bandodkar AJ, Wang J (2014) Non-invasive wearable electrochemical sensors: a review. Trends Biotechnol. 32:363-371

8. Scott, R. T. et al. Beyond Low Earth Orbit: Biomonitoring, Artificial Intelligence, and Precision Space Health.

9. Sokolov AN, Roberts ME, Bao Z (2009) Fabrication of low-cost electronic biosensors. Mater. Today 12:12-20

10. Roberts ME, Sokolov AN, Bao Z (2009) Material and device considerations for organic thin-film transistor sensors. J. Mater. Chem. 19:3351-3363

11. Kubota R, Sasaki Y, Minamiki T, Minami T (2019) Chemical Sensing Platforms Based on Organic Thin-Film Transistors Functionalized with Artificial Receptors. ACS Sensors 4:2571-2587

12. N Wang A Yang Y Fu Y Li F Yan 2019 Functionalized Organic Thin Film Transistors for Biosensing Acc. Chem. Res. https://doi. org/10.1021/acs.accounts.8b00448

13. Girgis A, Lambert S, Johnson C, Waller A, Currow D (2013) Physical, psychosocial, relationship, and economic burden of caring for people with cancer: A review. J. Oncol. Pract. 9:197-202

14. Sandbhor Gaikwad, P. \& Banerjee, R. Advances in point-of-care diagnostic devices in cancers. Analyst vol. 143 1326-1348 (2018).

15. Rais-Bahrami S et al (2015) Diagnostic value of biparametric magnetic resonance imaging (MRI) as an adjunct to prostatespecific antigen (PSA)-based detection of prostate cancer in men without prior biopsies. BJU Int. 115:381-388

16. Hambrock $\mathrm{T}$ et al (2010) Magnetic Resonance Imaging Guided Prostate Biopsy in Men With Repeat Negative Biopsies and Increased Prostate Specific Antigen. J. Urol. 183:520-528

17. Kuhl CK et al (2017) Abbreviated biparametric prostate MR imaging in men with elevated prostate-specific antigen. Radiology 285:493-505
18. Turkbey B et al (2013) Prostate cancer: Can multiparametric mr imaging help identify patients who are candidates for active surveillance? Radiology 268:144-152

19. Cimitan $\mathrm{M}$ et al (2006) [18F]fluorocholine PET/CT imaging for the detection of recurrent prostate cancer at PSA relapse: Experience in 100 consecutive patients. Eur. J. Nucl. Med. Mol. Imaging 33:1387-1398

20. Sarkar S, Das S (2016) A Review of Imaging Methods for Prostate Cancer Detection. Biomed. Eng. Comput. Biol. 7:1

21. Fortuin, A., De Rooij, M., Zamecnik, P., Haberkorn, U. \& Barentsz, J. Molecular and Functional Imaging for Detection of Lymph Node Metastases in Prostate Cancer. Int. J. Mol. Sci. 2013, Vol. 14, Pages 13842-13857 14, 13842-13857 (2013).

22. Zani A, Laschi S, Mascini M, Marrazza G (2011) A New Electrochemical Multiplexed Assay for PSA Cancer Marker Detection. Electroanalysis 23:91-99

23. Mani V, Chikkaveeraiah BV, Patel V, Gutkind JS, Rusling JF (2009) Ultrasensitive immunosensor for cancer biomarker proteins using gold nanoparticle film electrodes and multienzyme-particle amplification. ACS Nano 3:585-594

24. Yu X et al (2006) Carbon nanotube amplification strategies for highly sensitive immunodetection of cancer biomarkers. J. Am. Chem. Soc. 128:11199-11205

25. Sardesai, N., Pan, S. \& Rusling, J. Electrochemiluminescent immunosensor for detection of protein cancer biomarkers using carbon nanotube forests and [Ru-(bpy)3] 2+-doped silica nanoparticles. Chem. Commun. 0, 4968-4970 (2009).

26. Turkbey B et al (2016) Multiparametric prostate magnetic resonance imaging in the evaluation of prostate cancer. CA. Cancer J. Clin. 66:326-336

27. Wang, T. D., Chen, Y. \& Ronald, J. A. A novel approach for assessment of prostate cancer aggressiveness using survivindriven tumour-activatable minicircles. Gene Ther. $2019265 \mathbf{2 6}$, 177-186 (2019).

28. Hussain $\mathrm{T}$ et al (2016) Surgical molecular navigation with ratiometric activatable cell penetrating peptide for intraoperative identification and resection of small salivary gland cancers. Head Neck $38: 715-723$

29. Johansson, G. et al. Monitoring circulating tumor-DNA during surgical treatment in patients with gastrointestinal stromal tumors. Mol. Cancer Ther. molcanther.0403.2021 (2021) https://doi.org/ 10.1158/1535-7163.MCT-21-0403.

30. Kinross JM, Holmes E, Darzi AW, Nicholson JK (2011) Metabolic phenotyping for monitoring surgical patients. Lancet 377:1817-1819

31. Sobngwi E et al (2010) Continuous interstitial glucose monitoring in non-diabetic subjects with end-stage renal disease undergoing maintenance haemodialysis. Diabetes Res. Clin. Pract. 90:22-25

32. Zelnick LR et al (2020) Continuous Glucose Monitoring and Use of Alternative Markers To Assess Glycemia in Chronic Kidney Disease. Diabetes Care 43:2379-2387

33. Block, C. D., Manuel-y-Keenoy, B., Rogiers, P., Jorens, P. \& Gaal, L. Van. Glucose Control and Use of Continuous Glucose Monitoring in the Intensive Care Unit: A Critical Review. Curr. Diabetes Rev. 4, 234-244 (2008).

34. Butterfield, D. A. \& Halliwell, B. Oxidative stress, dysfunctional glucose metabolism and Alzheimer disease. Nat. Rev. Neurosci. 2019203 20, 148-160 (2019).

35. Peeks F et al (2021) A retrospective in-depth analysis of continuous glucose monitoring datasets for patients with hepatic glycogen storage disease: Recommended outcome parameters for glucose management. J. Inherit. Metab. Dis. 44:1136-1150

36. Chuang $\mathrm{H}$ et al (2008) Pilot studies of transdermal continuous glucose measurement in outpatient diabetic patients and in patients during and after cardiac surgery. J. Diabetes Sci. Technol. 2:595-602 
37. Chetrit $M$ et al (2020) Imaging-Guided Therapies for Pericardial Diseases. JACC Cardiovasc. Imaging 13:1422-1437

38. Namikawa, T., Sato, T. \& Hanazaki, K. Recent advances in nearinfrared fluorescence-guided imaging surgery using indocyanine green. Surg. Today 20154512 45, 1467-1474 (2015).

39. Zghaib T et al (2018) Standard Ablation Versus Magnetic Resonance Imaging-Guided Ablation in the Treatment of Ventricular Tachycardia. Circ. Arrhythmia Electrophysiol. 11:5973

40. Mauri, G. et al. Technical success, technique efficacy and complications of minimally-invasive imaging-guided percutaneous ablation procedures of breast cancer: A systematic review and meta-analysis. Eur. Radiol. 2016278 27, 3199-3210 (2017).

41. Wilson MS, Nie W (2006) Multiplex Measurement of Seven Tumor Markers Using an Electrochemical Protein Chip. Anal. Chem. 78:6476-6483

42. Wu, J. et al. Lab-on-a-chip platforms for detection of cardiovascular disease and cancer biomarkers. Sensors (Switzerland) vol. 172934 (2017).

43. Wu AM, Olafsen T (2008) Antibodies for molecular imaging of cancer. Cancer J. 14:191-197

44. Warram JM et al (2014) Antibody-based imaging strategies for cancer. Cancer Metastasis Rev. 33:809-822

45. Qi L et al (2020) Simultaneous Detection of Multiple Tumor Markers in Blood by Functional Liquid Crystal Sensors Assisted with Target-Induced Dissociation of Aptamer. Anal. Chem. 92:3867-3873

46. Yang C et al (2014) Multiplexed and Amplified Electronic Sensor for the Detection of MicroRNAs from Cancer Cells. Anal. Chem. 86:11913-11918

47. Zeng Z et al (2014) Specific and Sensitive Tumor Imaging Using Biostable Oligonucleotide Aptamer Probes. Theranostics 4:945

48. Chen, K. H. et al. c-erbB-2 sensing using AlGaN/GaN high electron mobility transistors for breast cancer detection. Appl. Phys. Lett. 92, 192103 (2008).

49. Goldenberg DM, Nabi HA (1999) Breast cancer imaging with radiolabeled antibodies. Semin. Nucl. Med. 29:41-48

50. Stipsanelli E, Valsamaki P (2005) Monoclonal antibodies: old and new trends in breast cancer imaging and therapeutic approach. Hell. J. Nucl. Med. 8:103-108

51. Li X et al (2020) Detection of Circulating Tumor Cells in Breast Cancer Patients by Nanopore Sensing with Aptamer-Mediated Amplification. ACS Sensors 5:2359-2366

52. Liu M et al (2018) An Aptamer-Based Probe for Molecular Subtyping of Breast Cancer. Theranostics 8:5772

53. Li P, Zhang B, Cui T (2015) Towards intrinsic graphene biosensor: A label-free, suspended single crystalline graphene sensor for multiplex lung cancer tumor markers detection. Biosens. Bioelectron. 72:168-174

54. Roointan A et al (2019) Early detection of lung cancer biomarkers through biosensor technology: A review. J. Pharm. Biomed. Anal. 164:93-103

55. Machac J, Krynyckyi B, Kim C (2002) Peptide and antibody imaging in lung cancer. Semin. Nucl. Med. 32:276-292

56. Breitz HB, Sullivan K, Nelp WB (1993) Imaging lung cancer with radiolabeled antibodies. Semin. Nucl. Med. 23:127-132

57. Huang X et al (2021) CKAP4 Antibody-Conjugated Si Quantum Dot Micelles for Targeted Imaging of Lung Cancer. Nanoscale Res. Lett. 16:1-12

58. Khatoon $\mathrm{Z}$ et al (2020) Feasibility study of doped $\mathrm{SnO} 2$ nanomaterial for electronic nose towards sensing biomarkers of lung cancer. J. Mater. Sci. Mater. Electron. 31:15751-15763

59. Ambrosini $\mathrm{V}$ et al (2012) PET/CT imaging in different types of lung cancer: An overview. Eur. J. Radiol. 81:988-1001

60. Mishra, S. et al. Tailored Biofunctionalized Biosensor for the Label-Free Sensing of Prostate-Specific Antigen. ACS Appl. Bio Mater. 2020, (2020).
61. Chen S et al (2019) Microfluidic Device Directly Fabricated on Screen-Printed Electrodes for Ultrasensitive Electrochemical Sensing of PSA. Nanoscale Res. Lett. 14:1-7

62. Lerner MB et al (2012) Hybrids of a genetically engineered antibody and a carbon nanotube transistor for detection of prostate cancer biomarkers. ACS Nano 6:5143-5149

63. Bander, N. H. Technology Insight: monoclonal antibody imaging of prostate cancer. Nat. Clin. Pract. Urol. 2006 34 3, 216-225 (2006).

64. Wiehr S et al (2014) Pharmacokinetics and PET imaging properties of two recombinant anti-PSMA antibody fragments in comparison to their parental antibody. Prostate 74:743-755

65. Savory N, Abe K, Sode K, Ikebukuro K (2010) Selection of DNA aptamer against prostate specific antigen using a genetic algorithm and application to sensing. Biosens. Bioelectron. 26:1386-1391

66. Zheng, G., Patolsky, F., Cui, Y., Wang, W. U. \& Lieber, C. M. Multiplexed electrical detection of cancer markers with nanowire sensor arrays. Nat. Biotechnol. 20052310 23, 1294-1301 (2005).

67. Wang AZ et al (2008) Superparamagnetic Iron Oxide Nanoparticle-Aptamer Bioconjugates for Combined Prostate Cancer Imaging and Therapy. ChemMedChem 3:1311

68. Abbasy, L., Mohammadzadeh, A., Hasanzadeh, M. \& Razmi, N. Development of a reliable bioanalytical method based on prostate specific antigen trapping on the cavity of molecular imprinted polymer towards sensing of PSA using binding affinity of PSAMIP receptor: A novel biosensor. J. Pharm. Biomed. Anal. 188, 113447 (2020).

69. Sciarra A et al (2011) Advances in Magnetic Resonance Imaging: How They Are Changing the Management of Prostate Cancer. Eur. Urol. 59:962-977

70. Altintas Z, Fakanya WM, Tothill IE (2014) Cardiovascular disease detection using bio-sensing techniques. Talanta 128:177-186

71. Justino CIL et al (2013) Disposable immunosensors for C-reactive protein based on carbon nanotubes field effect transistors. Talanta 108:165-170

72. Sanz, J. \& Fayad, Z. A. Imaging of atherosclerotic cardiovascular disease. Nat. 20084517181 451, 953-957 (2008).

73. Willner I, Zayats M (2007) Electronic Aptamer-Based Sensors. Angew. Chemie Int. Ed. 46:6408-6418

74. Kumar V et al (2016) Nanostructured aptamer-functionalized black phosphorus sensing platform for label-free detection of myoglobin, a cardiovascular disease biomarker. ACS Appl. Mater. Interfaces 8:22860-22868

75. Nakatsuka MA, Mattrey RF, Esener SC, Cha JN, Goodwin AP (2012) Aptamer-Crosslinked Microbubbles: Smart Contrast Agents for Thrombin-Activated Ultrasound Imaging. Adv. Mater. 24:6010-6016

76. Gupta S, Sharma A, Verma RS (2020) Polymers in biosensor devices for cardiovascular applications. Curr. Opin. Biomed. Eng. 13:69-75

77. Sogbein, O. O. et al. New SPECT and PET radiopharmaceuticals for imaging cardiovascular disease. Biomed Res. Int. 2014, (2014).

78. Jaffer FA, Libby P, Weissleder R (2007) Molecular imaging of cardiovascular disease. Circulation 116:1052-1061

79. Kim K, Lee CH, Park CB (2020) Chemical sensing platforms for detecting trace-level Alzheimer's core biomarkers. Chem. Soc. Rev. 49:5446-5472

80. Lien TTN, Takamura Y, Tamiya E, Vestergaard MC (2015) Modified screen printed electrode for development of a highly sensitive label-free impedimetric immunosensor to detect amyloid beta peptides. Anal. Chim. Acta 892:69-76

81. Kim, K. et al. Clinically accurate diagnosis of Alzheimer's disease via multiplexed sensing of core biomarkers in human plasma. Nat. Commun. 2020111 11, 1-9 (2020). 
82. Viola, K. L. et al. Towards non-invasive diagnostic imaging of early-stage Alzheimer's disease. Nat. Nanotechnol. 2014101 10, 91-98 (2014).

83. van Dyck CH (2018) Anti-Amyloid- $\beta$ Monoclonal Antibodies for Alzheimer's Disease: Pitfalls and Promise. Biol. Psychiatry 83:311-319

84. Sehlin, D. et al. Antibody-based PET imaging of amyloid beta in mouse models of Alzheimer's disease. Nat. Commun. 2016717 , 1-11 (2016)

85. Reiman EM, Jagust WJ (2012) Brain imaging in the study of Alzheimer's disease. Neuroimage 61:505-516

86. Kutovyi, Y. et al. Amyloid-beta peptide detection via aptamerfunctionalized nanowire sensors exploiting single-trap phenomena. Biosens. Bioelectron. 154, 112053 (2020).

87. Farrar, C. T., William, C. M., Hudry, E., Hashimoto, T. \& Hyman, B. T. RNA Aptamer Probes as Optical Imaging Agents for the Detection of Amyloid Plaques. PLoS One 9, e89901 (2014).

88. Qin, J., Kim, S., Cho, M. \& Lee, Y. Hierarchical and ultra-sensitive amyloid beta oligomer sensor for practical applications. Chem. Eng. J. 401, 126055 (2020).

89. Choi $\mathrm{G}$ et al (2020) Multifunctional imaging of amyloid-beta peptides with a new gadolinium-based contrast agent in Alzheimer's disease. J. Ind. Eng. Chem. 83:214-223

90. Nordberg A (2004) PET imaging of amyloid in Alzheimer's disease. Lancet Neurol. 3:519-527

91. Vlassenko, A. G., Benzinger, T. L. S. \& Morris, J. C. PET amyloid-beta imaging in preclinical Alzheimer's disease. Biochim. Biophys. Acta - Mol. Basis Dis. 1822, 370-379 (2012).

92. A Leuzy et al 2021 Biomarker-Based Prediction of Longitudinal Tau Positron Emission Tomography in Alzheimer Disease JAMA Neurol. https://doi.org/10.1001/JAMANEUROL.2021.4654

93. Tokuraku, K., Marquardt, M. \& Ikezu, T. Real-Time Imaging and Quantification of Amyloid- $\beta$ Peptide Aggregates by Novel Quantum-Dot Nanoprobes. PLoS One 4, e8492 (2009).

94. Liu X, Liu J (2021) Biosensors and sensors for dopamine detection. View 2:20200102

95. Liang, Y., Guo, T., Zhou, L., Offenhäusser, A. \& Mayer, D. Label-Free Split Aptamer Sensor for Femtomolar Detection of Dopamine by Means of Flexible Organic Electrochemical Transistors. Materials (Basel). 13, (2020).

96. McConnell EM et al (2019) In Vivo Use of a Multi-DNA Aptamer-Based Payload/Targeting System to Study Dopamine Dysregulation in the Central Nervous System. ACS Chem. Neurosci. 10:371-383

97. Badgaiyan RD (2014) Imaging dopamine neurotransmission in live human brain. Prog. Brain Res. 211:165

98. Shen, L. H., Liao, M. H. \& Tseng, Y. C. Recent Advances in Imaging of Dopaminergic Neurons for Evaluation of Neuropsychiatric Disorders. J. Biomed. Biotechnol. 2012, (2012).

99. Schatz DG, Ji Y (2011) Recombination centres and the orchestration of V(D)J recombination. Nat Rev Immunol 11:251-263

100. Harding FA, Stickler MM, Razo J, DuBridge RB (2010) The immunogenicity of humanized and fully human antibodies: Residual immunogenicity resides in the CDR regions. MAbs 2:256-265

101. Xu, W., Wang, D., Li, D. \& Liu, C. C. Recent Developments of Electrochemical and Optical Biosensors for Antibody Detection. Int. J. Mol. Sci. 2020, Vol. 21, Page 134 21, 134 (2019).

102. Casalini $S$ et al (2015) Multiscale sensing of antibody-antigen interactions by organic transistors and single-molecule force spectroscopy. ACS Nano 9:5051-5062

103. Wujcik EK et al (2014) Antibody nanosensors: a detailed review. RSC Adv. 4:43725-43745

104. Gao, S., Guisán, J. M. \& Rocha-Martin, J. Oriented immobilization of antibodies onto sensing platforms - A critical review. Anal. Chim. Acta 1189, 338907 (2022).
105. Kaur S et al (2012) Recent trends in antibody-based oncologic imaging. Cancer Lett. 315:97-111

106. Lee $\mathrm{M}$ et al (2012) A novel biosensor based on hafnium oxide: Application for early stage detection of human interleukin-10. Sensors Actuators, B Chem. 175:201-207

107. Mälarstig A et al (2008) Raised interleukin-10 is an indicator of poor outcome and enhanced systemic inflammation in patients with acute coronary syndrome. Heart 94:724-729

108. Chen YW, Liu M, Kaneko T, McIntyre PC (2010) Atomic layer deposited hafnium oxide gate dielectrics for charge-based biosensors. Electrochem. Solid-State Lett. 13:G29

109. Xenaki, K. T., Oliveira, S. \& van Bergen en Henegouwen, P. M. P. Antibody or Antibody Fragments: Implications for Molecular Imaging and Targeted Therapy of Solid Tumors. Front. Immunol. 0, 1287 (2017).

110. Freise AC, Wu AM (2015) In vivo imaging with antibodies and engineered fragments. Mol. Immunol. 67:142-152

111. Bates, A. \& Power, C. A. David vs. Goliath: The Structure, Function, and Clinical Prospects of Antibody Fragments. Antibodies 2019, Vol. 8, Page 28 8, 28 (2019).

112. Boileau NT, Melville OA, Mirka B, Cranston R, Lessard BH (2019) P and N type copper phthalocyanines as effective semiconductors in organic thin-film transistor based DNA biosensors at elevated temperatures. RSC Adv. 9:2133-2142

113. Hammock ML, Knopfmacher O, Naab BD, Tok JB-H, Bao Z (2013) Investigation of Protein Detection Parameters Using Nanofunctionalized Organic Field-Effect Transistors. ACS Nano 7:3970-3980

114. Agarwal DK, Kandpal M, Surya SG (2019) Characterization and detection of cardiac Troponin-T protein by using 'aptamer' mediated biofunctionalization of $\mathrm{ZnO}$ thin-film transistor. Appl. Surf. Sci. 466:874-881

115. Kratschmer C, Levy M (2017) Effect of Chemical Modifications on Aptamer Stability in Serum. Nucleic Acid Ther. 27:335

116. Chen $\mathrm{H}$ et al (2016) A review on electronic bio-sensing approaches based on non-antibody recognition elements. Analyst 141:2335-2346

117. Song S, Wang L, Li J, Fan C, Zhao J (2008) Aptamer-based biosensors. TrAC. Trends Anal. Chem. 27:108-117

118. LR Schoukroun-Barnes et al 2016 Reagentless, Structure-Switching ElectrochemAptamer Based Sensors https://doi.org/10.1146/ annurev-anchem-071015-0414469,163-181

119. Harrad, L. El, Bourais, I., Mohammadi, H. \& Amine, A. Recent advances in electrochemical biosensors based on enzyme inhibition for clinical and pharmaceutical applications. Sensors (Switzerland) 18, 164 (2018).

120. Magliulo M et al (2013) Part per Trillion Label-Free Electronic Bioanalytical Detection. Anal. Chem. 85:3849-3857

121. Takao Someya, *,†,\|, Ananth Dodabalapur*, $\dagger$, $\dagger$, Howard E. Katz, ${ }^{*} \dagger$ and \& Bao $\dagger, Z$. Integration and Response of Organic Electronics with Aqueous Microfluidics. Langmuir 18, 5299-5302 (2002).

122. Mabeck, J. T. \& Malliaras, G. G. Chemical and biological sensors based on organic thin-film transistors. Anal. Bioanal. Chem. 2005 3842 384, 343-353 (2005).

123. Xin, N. et al. Concepts in the design and engineering of singlemolecule electronic devices. Nat. Rev. Phys. 201913 1, 211-230 (2019).

124. Lin, M. et al. Electrochemical detection of nucleic acids, proteins, small molecules and cells using a DNA-nanostructure-based universal biosensing platform. Nat. Protoc. 2016117 11, 1244-1263 (2016).

125. Yen, Y. K. \& Chiu, C. Y. A CMOS MEMS-based MembraneBridge Nanomechanical Sensor for Small Molecule Detection. Sci. Reports 2020101 10, 1-8 (2020). 
126. Wang X, Qin L, Zhou M, Lou Z, Wei H (2018) Nanozyme Sensor Arrays for Detecting Versatile Analytes from Small Molecules to Proteins and Cells. Anal. Chem. 90:11696-11702

127. Comeau ZJ et al (2019) On-the-Spot Detection and Speciation of Cannabinoids Using Organic Thin-Film Transistors. ACS Sensors 4:2706-2715

128. Li Z, Fang M, LaGasse MK, Askim JR, Suslick KS (2017) Colorimetric Recognition of Aldehydes and Ketones. Angew. Chemie 129:9992-9995

129. Cagle BS, Crawford RA, Doorn JA (2019) Biogenic AldehydeMediated Mechanisms of Toxicity in Neurodegenerative Disease. Curr. Opin. Toxicol. 13:16

130. Wang W, Wang C, Xu H, Gao Y (2020) Aldehyde dehydrogenase, liver disease and cancer. Int. J. Biol. Sci. 16:921-934

131. O’Brien, P., Siraki, A. \& Shangari, N. Aldehyde Sources, Metabolism, Molecular Toxicity Mechanisms, and Possible Effects on Human Health. https://doi.org/10.1080/10408440591002183 35, 609-662 (2008).

132. Alkedeh, O. \& Priefer, R. The Ketogenic Diet: Breath Acetone Sensing Technology. Biosensors 11, (2021).

133. Sgobbi LF, Razzino CA, Machado SAS (2016) A disposable electrochemical sensor for simultaneous detection of sulfamethoxazole and trimethoprim antibiotics in urine based on multiwalled nanotubes decorated with Prussian blue nanocubes modified screen-printed electrode. Electrochim. Acta 191:1010-1017

134. Sellmyer MA et al (2020) Imaging CAR T Cell Trafficking with eDHFR as a PET Reporter Gene. Mol. Ther. 28:42-51

135. Dang $T$ et al (2018) Hydrazo-CEST: Hydrazone-Dependent Chemical Exchange Saturation Transfer Magnetic Resonance Imaging Contrast Agents. Chem. - A Eur. J. 24:9148-9156

136. Robinson ER et al (2021) Minicircles for a two-step blood biomarker and PET imaging early cancer detection strategy. J. Control. Release 335:281-289

137. Ronald JA, Chuang HY, Dragulescu-Andrasi A, Horia SS, Gambhira SS (2015) Detecting cancers through tumor-activatable minicircles that lead to a detectable blood biomarker. Proc. Natl. Acad. Sci. U. S. A. 112:3068-3073

138. Dawson J et al (2018) Xanthine oxidase inhibition for the improvement of long-term outcomes following ischaemic stroke and transient ischaemic attack (XILO-FIST) - Protocol for a randomised double blind placebo-controlled clinical trial. Eur. Stroke J. 3:281-290

139. Liu D, Wang D, Yang W, Meng D (2017) Potential anti-gout constituents as xanthine oxidase inhibitor from the fruits of Stauntonia brachyanthera. Bioorganic Med. Chem. 25:3562-3566

140. Patoulias D et al (2021) Meta-Analysis Assessing the Effects of Allopurinol on Left Ventricular Mass and Other Indices of Left Ventricular Remodeling as Evaluated by Cardiac Magnetic Resonance Imaging. Am. J. Cardiol. 138:129-132

141. Hason S et al (2009) Simultaneous electrochemical monitoring of metabolites related to the xanthine oxidase pathway using a grinded carbon electrode. Anal. Chem. 81:4302-4307

142. El Harrad L, Amine A (2016) Amperometric biosensor based on prussian blue and nafion modified screen-printed electrode for screening of potential xanthine oxidase inhibitors from medicinal plants. Enzyme Microb. Technol. 85:57-63

143. Arakawa $\mathrm{T}$ et al (2016) Mouthguard biosensor with telemetry system for monitoring of saliva glucose: A novel cavitas sensor. Biosens. Bioelectron. 84:106-111

144. Li, Y. J. \& Lu, C. C. A Novel Scheme and Evaluations on a LongTerm and Continuous Biosensor Platform Integrated with a Dental Implant Fixture and Its Prosthetic Abutment. Sensors 2015, Vol. 15, Pages 24961-24976 15, 24961-24976 (2015).

145. Mannoor, M. S. et al. Graphene-based wireless bacteria detection on tooth enamel. Nat. Commun. 201231 3, 1-9 (2012).

146. Gao W et al (2016) Fully integrated wearable sensor arrays for multiplexed in situ perspiration analysis. Nature 529:509-514
147. Wang, C. et al. Monitoring of the central blood pressure waveform via a conformal ultrasonic device. Nat. Biomed. Eng. 2018292 , 687-695 (2018).

148. Rodelo, C. G. et al. Zinc associated nanomaterials and their intervention in emerging respiratory viruses: Journey to the field of biomedicine and biomaterials. Coord. Chem. Rev. 457, 214402 (2022).

149. Kim SJ, Choi SJ, Jang JS, Cho HJ, Kim ID (2017) Innovative Nanosensor for Disease Diagnosis. Acc. Chem. Res. 50:1587-1596

150. Choi SJ et al (2016) WO3 Nanofiber-Based Biomarker Detectors Enabled by Protein-Encapsulated Catalyst Self-Assembled on Polystyrene Colloid Templates. Small 12:911-920

151. Park, S. min et al. A mountable toilet system for personalized health monitoring via the analysis of excreta. Nat. Biomed. Eng. 202046 4, 624-635 (2020).

152. Miller, I. J. et al. Real-time health monitoring through urine metabolomics. npj Digit. Med. 201921 2, 1-9 (2019).

153. Choden, P., Seesaard, T., Dorji, U., Sriphrapradang, C. \& Kerdcharoen, T. Urine odor detection by electronic nose for smart toilet application. ECTI-CON 2017 - 2017 14th Int. Conf. Electr. Eng. Comput. Telecommun. Inf. Technol. 190-193 (2017) https://doi. org/10.1109/ECTICON.2017.8096205.

154. Usman F et al (2019) A Review of Biosensors for Non-Invasive Diabetes Monitoring and Screening in Human Exhaled Breath. IEEE Access 7:5963-5974

155. Risby TH, Solga SF (2006) Current status of clinical breath analysis. Appl. Phys. B Lasers Opt. 85:421-426

156. Sukul, P., Trefz, P., Kamysek, S., Schubert, J. K. \& Miekisch, W. Instant effects of changing body positions on compositions of exhaled breath. J. Breath Res. 9, 047105 (2015).

157. Sterer, N. \& Rosenberg, M. Origin, Diagnosis, and Management Breath Odors.

158. King, J. et al. Isoprene and acetone concentration profiles during exercise on an ergometer. J. Breath Res. 3, 027006 (2009).

159. Yuen LH, Saxena NS, Park HS, Weinberg K, Kool ET (2016) Dark Hydrazone Fluorescence Labeling Agents Enable Imaging of Cellular Aldehydic Load. ACS Chem. Biol. 11:2312-2319

160. Akam EA et al (2019) Improving the reactivity of hydrazine-bearing MRI probes for in vivo imaging of lung fibrogenesis. Chem. Sci. 11:224-231

161. Chen $\mathrm{HH}$ et al. (2017) Molecular imaging of oxidized collagen quantifies pulmonary and hepatic fibrogenesis. JCI Insight 2

162. Moon HG et al (2018) Hollow Pt-Functionalized SnO2 Hemipill Network Formation Using a Bacterial Skeleton for the Noninvasive Diagnosis of Diabetes. ACS Sensors 3:661-669

163. Salehi S, Nikan E, Khodadadi AA, Mortazavi Y (2014) Highly sensitive carbon nanotubes-SnO2 nanocomposite sensor for acetone detection in diabetes mellitus breath. Sensors Actuators, B Chem. 205:261-267

164. Bajtarevic A et al (2009) Noninvasive detection of lung cancer by analysis of exhaled breath. BMC Cancer 9:348

165. Herbig J, Titzmann T, Beauchamp J, Kohl I, Hansel A (2008) Buffered end-tidal (BET) sampling - A novel method for real-time breath-gas analysis. J. Breath Res. 2:37008

166. Güntner AT et al (2019) Breath Sensors for Health Monitoring. ACS Sensors 4:268-280

167. Schiffman JD, Fisher PG, Gibbs P (2015) Early detection of cancer: past, present, and future. Am Soc Clin Oncol Educ Book 57-65. https://doi.org/10.14694/EdBook_AM.2015.35.57

168. Kraniotou C, Karadima V, Bellos G, Tsangaris GT (2018) Predictive biomarkers for type 2 of diabetes mellitus: Bridging the gap between systems research and personalized medicine. J. Proteomics 188:59-62

169. Wouters, O. J., O’Donoghue, D. J., Ritchie, J., Kanavos, P. G. \& Narva, A. S. Early chronic kidney disease: diagnosis, management 
and models of care. Nat. Rev. Nephrol. 2015118 11, 491-502 (2015).

170. Chiappin S, Antonelli G, Gatti R, De Palo EF (2007) Saliva specimen: A new laboratory tool for diagnostic and basic investigation. Clinica Chimica Acta 383:30-40

171. Chicharro JL, Lucía A, Pérez M, Vaquero AF, Ureña R (1998) Saliva composition and exercise. Sports Med 26:17-27

172. Malon, R. S. P., Sadir, S., Balakrishnan, M. \& Córcoles, E. P. Saliva-Based Biosensors: Noninvasive Monitoring Tool for Clinical Diagnostics. BioMed Res Int vol. 2014 (2014).

173. Kalk WWI et al (2002) Sialometry and sialochemistry: A noninvasive approach for diagnosing Sjögren's syndrome. Ann. Rheum. Dis. 61:137-144

174. Aluoch AO, Sadik OA, Bedi G (2005) Development of an oral biosensor for salivary amylase using a monodispersed silver for signal amplification. Anal. Biochem. 340:136-144

175. Kwan RCH et al (2005) Amperometric biosensor for determining human salivary phosphate. Anal. Biochem. 343:263-267

176. Yamaguchi $\mathrm{M}$ et al (2004) Non-invasive monitoring of gingival crevicular fluid for estimation of blood glucose level. Med. Biol. Eng. Comput. 42:322-327

177. Guilbault GG, Palleschi G, Lubrano G (1995) Non-invasive biosensors in clinical analysis. Biosens. Bioelectron. 10:379-392

178. Ballesta Claver, J., Valencia Mirón, M. C. \& Capitán-Vallvey, L. F. Disposable electrochemiluminescent biosensor for lactate determination in saliva. Analyst 134, 1423-1432 (2009).

179. Palleschi G, Faridnia MH, Lubrano GJ, Guilbault GG (1991) Determination of lactate in human saliva with an electrochemical enzyme probe. Anal. Chim. Acta 245:151-157

180. Piechotta G, Albers J, Hintsche R (2005) Novel micromachined silicon sensor for continuous glucose monitoring. Biosens. Bioelectron. 21:802-808

181. Valdés-Ramírez G et al (2014) Non-invasive mouthguard biosensor for continuous salivary monitoring of metabolites. Analyst 139:1632-1636

182. Zheng C-M, Lu K-C, Wu C-C, Hsu Y-H, Lin Y-F (2011) Association of Serum Phosphate and Related Factors in ESRD-Related Vascular Calcification. Int. J. Nephrol. 2011:1-8

183. Shetty V, Zigler C, Robles TF, Elashoff D, Yamaguchi M (2011) Developmental validation of a point-of-care, salivary $\alpha$-amylase biosensor. Psychoneuroendocrinology 36:193-199

184. Kalman DS et al (2008) Effect of a proprietary Magnolia and Phellodendron extract on stress levels in healthy women: A pilot, double-blind, placebo-controlled clinical trial. Nutr. J. 7:1-6

185. Sun K, Ramgir N, Bhansali S (2008) An immunoelectrochemical sensor for salivary cortisol measurement. Sensors Actuators, B Chem. 133:533-537

186. Dhull N, Kaur G, Gupta V, Tomar M (2019) Highly sensitive and non-invasive electrochemical immunosensor for salivary cortisol detection. Sensors Actuators, B Chem. 293:281-288

187. Mitchell JS, Lowe TE (2009) Ultrasensitive detection of testosterone using conjugate linker technology in a nanoparticle-enhanced surface plasmon resonance biosensor. Biosens. Bioelectron. 24:2177-2183

188. Aydın M, Aydın EB, Sezgintürk MK (2018) A highly selective electrochemical immunosensor based on conductive carbon black and star PGMA polymer composite material for IL-8 biomarker detection in human serum and saliva. Biosens. Bioelectron. 117:720-728

189. Sánchez-Tirado E et al (2017) Electrochemical immunosensor for simultaneous determination of interleukin-1 beta and tumor necrosis factor alpha in serum and saliva using dual screen printed electrodes modified with functionalized double-walled carbon nanotubes. Anal. Chim. Acta 959:66-73

190. Choudhary M et al (2016) CD 59 Targeted Ultrasensitive Electrochemical Immunosensor for Fast and Noninvasive Diagnosis of Oral Cancer. Electroanalysis 28:2565-2574

191. Sánchez-Tirado, E., González-Cortés, A., Yáñez-Sedeño, P. \& Pingarrón, J. M. Electrochemical immunosensor for the determination of the cytokine interferon gamma (IFN- $\gamma$ ) in saliva. Talanta 211, 120761 (2020).

192. Fabiani, L. et al. Magnetic beads combined with carbon blackbased screen-printed electrodes for COVID-19: A reliable and miniaturized electrochemical immunosensor for SARS-CoV-2 detection in saliva. Biosens. Bioelectron. 171, 112686 (2021)

193. McArdle WD et al (2006) Essentials of exercise physiology. Lippincott Williams \& Wilkins, United Kingdom

194. Montain SJ, Cheuvront SN, Lukaski HC (2007) Sweat mineralelement responses during $7 \mathrm{~h}$ of exercise-heat stress. Int. J. Sport Nutr. Exerc. Metab. 17:574-582

195. Briganti, S. et al. Application of Sebum Lipidomics to Biomarkers Discovery in Neurodegenerative Diseases. Metab. 2021, Vol. 11, Page 819 11, 819 (2021).

196. Sinclair, E. et al. Metabolomics of sebum reveals lipid dysregulation in Parkinson's disease. Nat. Commun. 2021121 12, 1-9 (2021).

197. Chung, M., Fortunato, G. \& Radacsi, N. Wearable flexible sweat sensors for healthcare monitoring: A review. J Royal Soc Interface vol. 16 (2019)

198. Kim J, Campbell AS, de Ávila BEF, Wang J (2019) Wearable biosensors for healthcare monitoring. Nat Biotechnol 37:389-406

199. Kwong GA et al (2013) Mass-encoded synthetic biomarkers for multiplexed urinary monitoring of disease. Nat. Biotechnol. 31:63

200. Gadalla, A. A. H. et al. Identification of clinical and urine biomarkers for uncomplicated urinary tract infection using machine learning algorithms. Sci. Reports 201991 9, 1-11 (2019).

201. Grayson, K., Gregory, E., Khan, G. \& Guinn, B.-A. Urine Biomarkers for the Early Detection of Ovarian Cancer - Are We There Yet?: https://doi.org/10.1177/1179299X19830977 11, 1179299X1983097 (2019).

202. Koyner JL et al (2010) Urinary Biomarkers in the Clinical Prognosis and Early Detection of Acute Kidney Injury. Clin. J. Am. Soc. Nephrol. 5:2154

203. Jin K et al (2017) Intensive Monitoring of Urine Output Is Associated With Increased Detection of Acute Kidney Injury and Improved Outcomes. Chest 152:972-979

204. Lachance P et al (2017) Association between e-alert implementation for detection of acute kidney injury and outcomes: a systematic review. Nephrol. Dial. Transplant. 32:265-272

205. Park, S. min, Ge, T. J., Won, D. D., Lee, J. K. \& Liao, J. C. Digital biomarkers in human excreta. Nat. Rev. Gastroenterol. Hepatol. 18, (2021).

206. Farkas K, Hillary LS, Malham SK, McDonald JE, Jones DL (2020) Wastewater and public health: the potential of wastewater surveillance for monitoring COVID-19. Curr. Opin. Environ. Sci. Heal. 17:14-20

207. Arroyo-Currás N et al (2017) Real-time measurement of small molecules directly in awake, ambulatory animals. Proc. Natl. Acad. Sci. 114:645-650

Publisher's Note Springer Nature remains neutral with regard to jurisdictional claims in published maps and institutional affiliations. 